\title{
Retinal Vascular Network Topology Reconstruction and Artery/Vein Classification via Dominant Set Clustering
}

\author{
Yitian Zhao, Jianyang Xie, Huaizhong Zhang, Yalin Zheng, Yifan Zhao, Hong Qi, Yangchun Zhao, Pan Su*, \\ Jiang Liu and Yonghuai Liu*
}

\begin{abstract}
The estimation of vascular network topology in complex networks is important in understanding the relationship between vascular changes and a wide spectrum of diseases. Automatic classification of the retinal vascular trees into arteries and veins is of direct assistance to the ophthalmologist in terms of diagnosis and treatment of eye disease. However, it is challenging due to their projective ambiguity and subtle changes in appearance, contrast and geometry in the imaging process. In this paper, we propose a novel method that is capable of making the artery/vein (A/V) distinction in retinal color fundus images based on vascular network topological properties. To this end, we adapt the concept of dominant set clustering and formalize the retinal blood vessel topology estimation and the A/V classification as a pairwise clustering problem. The graph is constructed through image segmentation, skeletonization and identification of significant nodes. The edge weight is defined as the inverse Euclidean distance between its two end points in the feature space of intensity, orientation, curvature, diameter, and entropy. The reconstructed vascular network is classified into arteries and veins based on their intensity and morphology. The proposed approach has been applied to five public databases, INSPIRE, IOSTAR, VICAVR, DRIVE and WIDE, and achieved high accuracies of $95.1 \%, 94.2 \%, 93.8 \%, 91.1 \%$, and $91.0 \%$, respectively. Furthermore, we have made manual annotations of the blood vessel topologies for INSPIRE, IOSTAR, VICAVR, and DRIVE datasets, and these annotations are released for public access so as to facilitate researchers in the community.
\end{abstract}

Index Terms-Retinal images, dominant set clustering, blood vessel, vascular topology, Artery/vein classification.

This work was supported by National Science Foundation Program of China (61601029), Chinese Postdoctoral Science Foundation (2019M652156), Zhejiang Provincial Natural Science Foundation (LZ19F010001), Ningbo Natural Science Foundation (2018A610055), Grant of Ningbo 3315 Innovation Team. (Corresponding authors: Pan Su and Yonghuai Liu)

Y. Zhao, J. Xie, P. Su, and J. Liu are with Cixi Instuitue of Biomedical Engineering, Ningbo Institute of Industrial Technology, Chinese Academy of Sciences, China; (supan@nimte.ac.cn)

H. Zhang and Y. Liu are with Department of Computer Science, Edge Hill University, UK; (yonghuai.liu@edgehill.ac.uk)

Y. Zheng is with Department of Eye and Vision Science, University of Liverpool, UK;

YF. Zhao is with School of Aerospace, Transport and Manufacturing, Cranfield University, UK;

H. Qi is with Department of Ophthalmology, Peking University Third Hospital, China;

YC. Zhao is with the Second Affiliated Hospital of Zhejiang Chinese Medical University, China;

J. Liu is also with Department of Computer Science and Engineering, Southern University of Science and Technology, China;

P. Su is also with School of Control and Computer Engineering, North China Electric Power University, China.

\section{INTRODUCTION}

The retinal blood vascular network is the only vascular network of the human body that is visible to a non-invasive imaging approach. In consequence, automated analysis of retinal vascular structure is the most common way to support examination, diagnosis and treatment of many diseases [1], [2], such as diabetic retinopathy, hypertension and other cardiovascular diseases [3], [4]. Retinal arteriolar constriction, or arteriovenous nicking, significant dilatation and elongation of main arteries and veins, or vascular caliber and tortuosity change are frequently associated with the aforementioned diseases [5]. It is crucial to identify and distinguish the structures of individual blood vessels from the entire blood vessel network in a given fundus image [6]. In particular, the Arteriolar-to-Venular Ratio (AVR) is considered to be an important characteristic sign that quantifies the severity of a wide spectrum of diseases [1], [7], [8], for example, low AVR - i.e., narrowing of arteries and widening of veins - is a direct biomarker for diabetic retinopathy. By contrast, a high AVR has been associated with higher cholesterol levels and inflammatory markers [9]. For these clinical observations, it would be of direct benefit if the retinal vascular network could be distinguished anatomically into different blood vessel branches, or separated into arteries and veins.

In practice, ophthalmologists use color and morphological information to discriminate between arteries and veins, since the arteries contain more oxygen and appear brighter than the veins, and thinner than neighboring veins [10]. These features of the retinal vasculature are usually captured by fundus photography, due to its lower cost and ease of use. Manual classification of retinal blood vessels is time consuming and subject to human errors. Therefore, an automatic vascular tracing method for topology reconstruction, or classification of blood vessels as arteries and veins, is highly desirable in seeking to overcome time constraints and avoid human errors. This calls for precise description of the vascular structure in terms of its color, topological, geometrical and morphological properties as derived from retinal images. However, it is highly challenging to discriminate arteries from veins, or perform any other measurement of interest (e.g., topology estimation), if the blood vessel widths are small, even after accurately segmenting the vasculature from the given fundus images. For example, existing methods often fail to trace correctly when faced with the occurrence of bifurcation and crossover 
at junction points: (i) the bifurcation - different blood vessel segments are from one blood vessel tree, and (ii) crossovers two blood vessels overlap due to the projection of a 3D human eye to a $2 \mathrm{D}$ fundus image. These intersections usually lead to difficulty in predicting whether given blood vessels contacting a junction belong to the same blood vessel tree, or a different tree, due to their projective ambiguity and subtle changes in appearance, contrast and geometry in the imaging process.

To address these problems, we propose a novel topology estimation and arteries/veins (A/V) classification method by adapting the concept of dominant sets clustering (DOS), in which a dominant set is used to represent a vascular tree. The novel method includes three main steps: (i) we reconceptualize our previous work [11] and extend it for both the retinal blood vessel topology estimation and classification, (ii) formalize them as a pairwise clustering problem, and (iii) validate the proposed method over five public retinal datasets. DOS is a graph-theoretic approach, and is a well-known generalization of the notion of maximal cliques to an edge-weighted graph. It has been proven to work well in data clustering and image segmentation [12]-[14]. We aim to classify the entire retinal blood vessel network, not just the most prominent blood vessels, with a view to clarifying the underlying topology - how different blood vessels are anatomically connected to each other -and to identify and distinguish the structure of individual blood vessels from the entire blood vessel network. To classify the reconstructed vascular network into arteries and veins, we consider domain-specific knowledge about how they perfuse the retina, including angular, morphological, and textural properties of all blood vessel segments of the junction. We also take into account the fact that arteries and veins usually alternate near the optic disc.

The main contributions of this paper are as follows:

- The concept of dominant set clustering has been introduced to tackle the challenging problem of vasculature analysis, and proved to be an effective way of addressing the problem of tracing crossovers.

- The proposed method can split the entire blood vessel graph into several individual branches as subtrees, and is capable of demonstrating how different blood vessels are anatomically connected to each other. In addition, the $\mathrm{A} / \mathrm{V}$ classification is undertaken on the topology-assigned blood vessel network, rather than the entire blood vessel segments.

- The proposed method has been validated quantitatively using five publicly accessible datasets, with promising results. In addition, the manual annotations of blood vessel topologies of four datasets were established as the ground truth, and have been released for public acces 11 .

The remainder of this paper is organized as follows. In Section II, the existing methods for retinal A/V classification and topology reconstruction are briefly reviewed. Section III details the proposed blood vessel topology estimation and A/V classification method. Section IV introduces the datasets, ground truth and evaluation metrics we will use. In Sections $\mathrm{V}$ and VI, we present the experimental results and discuss

1http://imed.nimte.ac.cn/vetovessel-topology-groundtruth.html the effectiveness, robustness, and efficiency respectively of the proposed method. Finally, in Section VII we draw conclusions, and indicate directions for future work.

\section{RELATED WORKS}

In the past decade, extensive work has been carried out on automatic retinal blood vessel segmentation [15]-[19]. However, automated $\mathrm{A} / \mathrm{V}$ classification and vascular topology reconstruction are still understudied, despite their significance to understanding the structure and distribution of the blood vessels. The majority of existing blood vessel classification methods make use of machine learning techniques, using local features and adding structural information from the vascular tree.

Martinez-Perez et al. [20] proposed a semi-automatic retinal blood vessel analysis method that is capable of measuring and quantifying the geometrical and topological properties. It requires a human expert first to classify a branch as either vein or artery. Kondermann et al. [21] classified blood vessels by utilizing the Support Vector Machines (SVM) and neural networks. Relevant features were extracted from the blood vessel profile and the regions of interest centred at the pixels on the blood vessel centerlines. Niemeijer et al. [1], [22] found the k-Nearest Neighbour $(\mathrm{kNN})$ classifier performing the best, by testing four different classifiers in the task of distinguishing between arteries and veins from a feature vector containing 27 elements. These elements were generated from blood vessel centerline pixels: a soft label was assigned to each centerline, implying the likelihood of the blood vessel being a vein. Mirsharif et al. [23] classified the blood vessels into arteries and veins by using multiple classifiers, and found that the Linear Discriminant Analysis (LDA) classifier had the best performance. Relan et al. [24] automatically classified retinal blood vessels as arteries or veins using color features and a Gaussian Mixture Model (GMM). Vazquez et al. [3] combined color-based clustering and blood vessel tracking to differentiate arteries from veins, and the tracking strategy based on the minimal path approach was employed to support the resulting classification by voting. Girard and Cheriet [25] trained a convolutional neural network $(\mathrm{CNN})$ for the task of assigning blood vessel pixels into arteries or veins. This approach propagated the blood vessel graph by using the minimum spanning tree. Huang et al. [26] introduced four new features to avoid distortions resulting from lightness inhomogeneity, and the accuracy of the A/V classification was improved by using the LDA classifier.

Due to demanding precise segmentation results in most of the existing methods, the ambiguity of small and midsized blood vessels makes the $\mathrm{A} / \mathrm{V}$ classification a very difficult computational task. Pathological conditions and intensity inhomogeneities further complicate the task of $\mathrm{A} / \mathrm{V}$ classification. More recently, the analysis of graphs extracted from the retinal blood vessel structure has been utilized to assist in blood vessel classification [9], [10], [27], [28]. This approach classifies the entire vascular tree by determining the type of all intersection pixels (graph nodes) and then assigning a label to each blood vessel segment, so as to reconstruct the underlying blood 


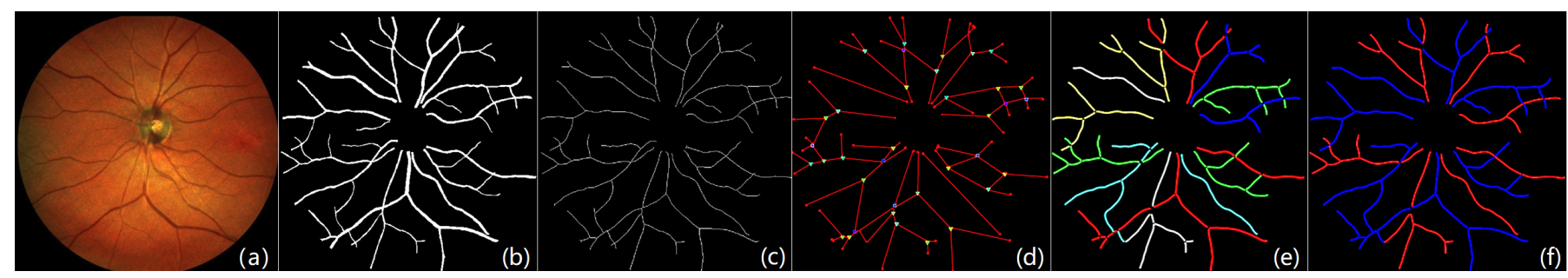

Fig. 1: Overview of the proposed method. (a) Original image. (b) Extracted blood vessels. (c) Skeletonized blood vessels. (d) Graph generated with significant nodes overlaid. (e) Estimated vascular network topology. (f) Classified arteries and veins: arteries shown in red, and veins in blue.

vessel topology with more accurate classification of small and midsized blood vessels. Rothaus et al. [27] presented an automated graph separation algorithm to distinguish between arteries and veins. Dashtbozorg et al. [9] proposed a method which first split the vascular graph into subgraphs by embedding the graph nodes and applying intensity features, and then, using LDA, assigned a label to each subgraph stating whether it was artery or vein. Joshi et al. [10], [28] separated their vascular graph into different subgraphs by using Dijkstra's shortestpath algorithm, then labeled each subgraph as either artery or vein using a fuzzy C-means clustering algorithm. Estrada et al. [5], [29] utilized a global likelihood model to capture the structural plausibility of each blood vessel, and employed a graph-theoretic method to estimate the overall blood vessel topology with domain-specific knowledge and applied three features, local growth, overlap and color, to accurately classify the A/V types. Lyu et al. [30] used a curvature orientation histogram to extract blood vessel landmarks from the blood vessel centerline, and separated different blood vessel trees by curvature orientation clustering. De et al. [31], [32] proposed a graph-theoretical approach to reconstruct the blood vessel network from topological information. They adapted the label propagation over directed graphs, and by this method the graph was partitioned into disjoint subgraphs. However, these topology reconstruction or subgraph estimation methods often fail to achieve accurate results due to the difficulty in identifying the presence of crossovers at blood vessel junctions, as the caliber, angular or other measurements are unreliable at points of abrupt change from one blood vessel to another.

\section{METHOD}

The proposed method adapts the dominant set clustering for retinal topology reconstruction and A/V classification, and the overview of the method is illustrated in Fig. 11 Fig. 1 1 a) shows an example retinal color fundus image from the public IOSTAR dataset, and Fig. 1.b) illustrates its extracted vasculature. Then a skeltonization step is applied to the extracted blood vessels, as shown in Fig. 11(c). A vessel graph is generated by removing the junction pixels from Fig. 1.(c), and Fig. 11.d) depicts the generated graph with significant nodes overlaid. The blood vessel network is separated into several individual tree branches in different colors, as shown in Fig. 1 (e). Finally, these tree branches are classified into two categories: arteries (red) and veins (blue). The generation of the graph, the separation and classification of the blood vessel network into arteries and veins are detailed below in Section III-A, Sections III-B and III-C, and Section III-D respectively.

\section{A. Graph generation}

Firstly, the optic disc is masked in order to avoid morphological complications due to the irregular and highly tortuous blood vessels at the optic disc: the superpixel-based optic disc segmentation [33] was utilized for its fast speed and effectiveness. Then the infinite perimeter active contour with hybrid region (IPACHR) method proposed in [34] is employed to automatically segment the retinal blood vessels for its effectiveness in detecting vessels with irregular and oscillatory boundaries, as shown in Fig. 1(b). An iterative morphology thinning operation [35] is finally performed on the extracted blood vessels to obtain a single-pixel-wide skeleton map, as shown in Fig. 1(c).

The vascular bifurcations, crossovers, and blood vessel ends (terminal points) may then be extracted from the skeleton map by locating intersection points (pixels with more than two neighbors) and terminal points (pixels with one neighbor). All the intersection points and their neighbors are then removed from the skeleton map, producing an image with clearly separated blood vessel segments. A blood vessel graph can be generated from this skeleton map by linking the first and last nodes in the same blood vessel segment. The generated graph will usually include some misrepresentations of the blood vessels: typical errors include node splitting, missing links and false links. Correction of these errors was achieved by employing the strategy proposed in [9], which considers the local vessel calibers and angles to reconnect the missing links and correct the false links. Fig. 1(d) shows the blood vessel graph, in which red dots indicate terminal points, green triangles bifurcations, and blue squares crossover points.

The intersections extracted from the skeleton map are critical to the final topology estimation. In the following, the details of partitioning the points in each intersection into different vascular segments are described. The analysis of the intersections is broken down into three categories, according to the number of points involved in each intersection: connecting points (2), bifurcation points $(3,4)$, and crossovers/meeting points $(3,4,5)$, where the number in the brackets after each category indicates the number of vascular segments 
connected to that intersection. Again, the method proposed by Dashtbozorg et al. [9] is used to handle the cases of nodes of degree 2. For the more complicated categories, nodes of degree 3 to 5 , a clustering method based on the dominant sets is proposed as follows.

The concept of dominant sets arises from the study of graph theory, by which a continuous formulation of the maximum clique problem is defined in [12]. An undirected graph $G$ with weighted edges is represented as $G=(V, E, \omega)$, where $V$ is a set of nodes, edge set $E \subseteq V \times V$ indicates all the connections of the relevant nodes, and $\omega: E \rightarrow \mathbb{R}^{+}$is the positive weight function. Fig. 2 2 shows an example of the edgeweighted graph extracted from a representative junction of a retinal blood vessel network.
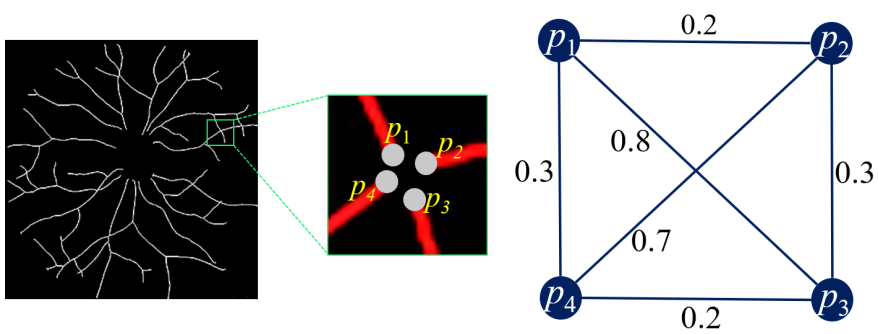

Fig. 2: An example of the edge-weighted graph.

In the context of vascular topology estimation and arteries/veins identification, $V$ is a set of extracted pixels from a retinal color fundus image and $\omega$ represents the similarity among the pixels in $V$. A $|V| \times|V|$ symmetric matrix $A=\left\{a_{i j}\right\}$ is used to represent the weighted graph $G$, which is named an adjacency matrix. The value of $a_{i j}$ is derived by a similarity measure defined in the feature space of the pixels. Here, we define $a_{i j}=0$ for $i=j$, which indicates that the generated graph $G$ does not include self-loop.

\section{B. Dominant sets}

A dominant set can be formally defined based on the values of similarity among the nodes in $V$. Let $S \subseteq V$ be a nonempty subset of nodes, $p_{i} \in S$ and $p_{j} \in S \backslash\left\{p_{i}\right\}$, where $S \backslash\left\{p_{i}\right\}$ indicates the nodes in the set $S$ excluding the node $p_{i}$. The relative similarity between $p_{i}$ and $p_{j}$ with respect to the average similarity between $p_{j}$ and its neighbours in $S \backslash\left\{p_{i}\right\}$ can be defined as:

$$
\phi_{S \backslash\left\{p_{i}\right\}}\left(p_{i}, p_{j}\right)=a_{i j}-\frac{1}{|S|-1} \sum_{p_{k} \in S \backslash\left\{p_{i}\right\}} a_{j k},
$$

where $|\cdot|$ denotes the cardinality of a set. For example, Fig. 2 depicts an edge-weighted graph generated from an intersection which is extracted from Fig. 1, where the weights of the edges indicate the similarity amongst four pixels $\left\{p_{1}, p_{2}, p_{3}, p_{4}\right\}$. Given $S=\left\{p_{1}, p_{2}, p_{3}\right\}$ we have: $\phi_{\left\{p_{1}, p_{3}\right\}}\left(p_{2}, p_{1}\right)=a_{21}-$ $\left(a_{11}+a_{13}\right) / 2=-0.2$ and $\phi_{\left\{p_{1}, p_{3}\right\}}\left(p_{2}, p_{3}\right)=a_{23}-\left(a_{31}+\right.$ $\left.a_{33}\right) / 2=-0.1$. It can be observed that $\phi_{S}\left(p_{i}, p_{j}\right)$ can be either positive or negative.

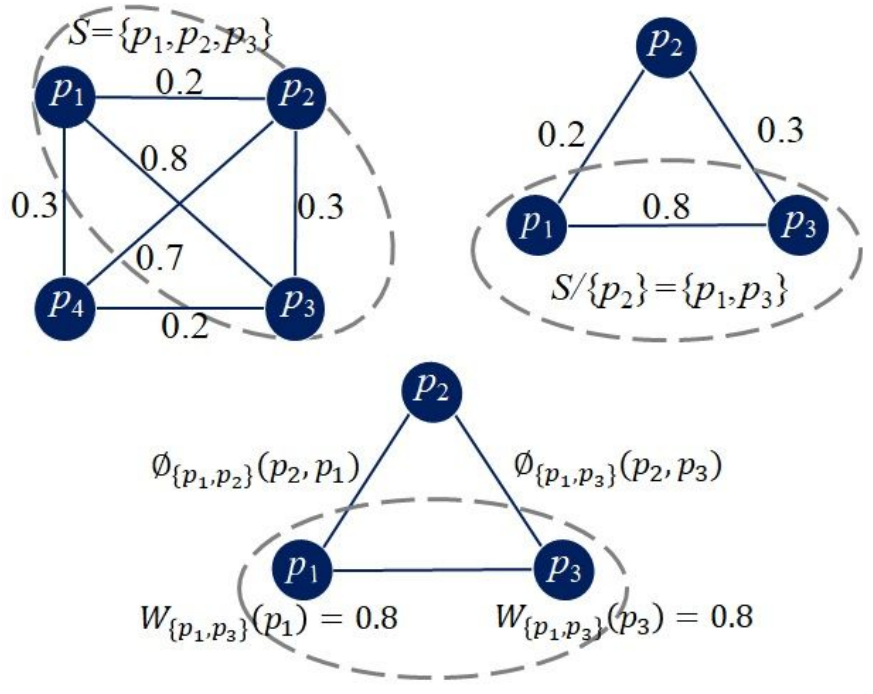

Fig. 3: An example of decomposed recursive calculation of similarity over a graph for dominant sets.

The weight $W_{S}\left(p_{i}\right)$ of $p_{i}$ with regard to $S$ is defined recursively as:

$W_{S}\left(p_{i}\right)= \begin{cases}1, & \text { if }|S|=1 \\ \sum_{p_{j} \in S \backslash\left\{p_{i}\right\}} \phi_{S \backslash\left\{p_{i}\right\}}\left(p_{i}, p_{j}\right) W_{S \backslash\left\{p_{i}\right\}}\left(p_{j}\right), & \text { otherwise. }\end{cases}$

where $W_{S}\left(p_{i}\right)$ expresses the similarity between node $p_{i}$ and the nodes of $S \backslash\left\{p_{i}\right\}$ with respect to the mutual similarity amongst the nodes in $S \backslash\left\{p_{i}\right\}$. Finally, the total weight of $S$ is calculated as $W(S)=\sum_{p_{i} \in S} W_{S}\left(p_{i}\right)$. Take the calculation of $W_{\left\{p_{1}, p_{3}, p_{4}\right\}}\left(p_{4}\right)$ as an example (shown in Fig. 3 , we have: $W_{\left\{p_{1}, p_{3}\right\}}\left(p_{1}\right)=W_{\left\{p_{1}, p_{3}\right\}}\left(p_{3}\right)=0.8, W_{\left\{p_{1}, p_{2}, p_{3}\right\}}\left(p_{2}\right)=$ $\phi_{\left\{p_{1}, p_{3}\right\}}\left(p_{2}, p_{1}\right) W_{\left\{p_{1}, p_{3}\right\}}\left(p_{1}\right)+\phi_{\left\{p_{1}, p_{3}\right\}}\left(p_{2}, p_{3}\right) W_{\left\{p_{1}, p_{3}\right\}}\left(p_{3}\right)$ $=-0.24$, and $W_{\left\{p_{1}, p_{3}, p_{4}\right\}}\left(p_{4}\right)=-0.24$.

Formally, a non-empty subset $S$ of nodes $V, S \subseteq V$ such that $W\left(S^{\prime}\right)>0$ for any non-empty subset $S^{\prime} \subseteq S$ is said to be a dominant set if:

$$
W_{S^{\prime}}\left(p_{i}\right)>0, \text { for all } p_{i} \in S^{\prime}
$$

and

$$
W_{S^{\prime} \cup\left\{p_{j}\right\}}\left(p_{j}\right)<0 \text {, for any } p_{j} \notin S^{\prime} .
$$

Take the graph shown in Fig. 3 as an example: $W\left(\left\{p_{1}, p_{3}\right\}\right)=$ $W_{\left\{p_{1}, p_{3}\right\}}\left(p_{1}\right)+W_{\left\{p_{1}, p_{3}\right\}}\left(p_{3}\right)=1.6, W\left(\left\{p_{1}\right\}\right)=W\left(\left\{p_{3}\right\}\right)=$ 1 , and $W_{\left\{p_{1}, p_{3}\right\}}\left(p_{1}\right)>0, W_{\left\{p_{1}, p_{3}\right\}}\left(p_{3}\right)>0, W_{\left\{p_{1}, p_{2}, p_{3}\right\}}\left(p_{2}\right)$ $<0, W_{\left\{p_{1}, p_{3}, p_{4}\right\}}\left(p_{4}\right)<0$. Therefore, $p_{1}$ and $p_{3}$ form a dominant set. Similarly, $p_{2}$ and $p_{4}$ form another dominant set.

In general, the weights of edges within the dominant set of an edge-weighted graph should be large, representing high internal homogeneity or similarity [12]. By contrast, the weights of edges which link to the dominant sets externally will be small. Therefore, the dominant set is a proper solution to identify branches of a vascular tree, because the similarity of two points from the same branch should be large within an intersection, while that of two points belonging to different branches should be small. 
Dominant sets can be identified by local solutions of a standard quadratic program:

$$
\begin{array}{ll}
\text { maximize }_{\mathbf{x}} & f(\mathbf{x})=\mathbf{x}^{\top} A \mathbf{x} \\
\text { subject to } & \mathbf{x} \in \Delta,
\end{array}
$$

where

$\Delta=\left\{\mathrm{x} \in \mathbb{R}^{|V|}: \sum_{i=1}^{|V|} x_{i}=1\right.$ and $x_{i} \geq 0$ for all $\left.i=1, \cdots,|V|\right\}$.

A strict local solution $\mathrm{x}^{*}$ of Eqn. (5) is named the weighted characteristic vector, where $x_{i}>0$ means that the node $p_{i}$ in question is in a dominant set of $G$. An effective optimization approach for solving Eqn. (5) is given by the replicator dynamics [13], [36]:

$$
x_{i}^{(t+1)}=x_{i}^{(t)} \frac{\left(A \mathbf{x}^{(t)}\right)_{i}}{\mathbf{x}^{(t)^{\top} A \mathbf{x}^{(t)}},}
$$

where $i=1,2, \cdots,|V|$, and $t$ denotes the iteration number with an initial value of zero. It has been proven that as $t$ in Eqn. (6) increases, for any initialization of $\mathbf{x}^{(0)} \in \Delta$, the trajectory remains in $\Delta$ and the objective function $f(\mathbf{x})$ in Eqn. (5) is either strictly increasing or constant. Therefore, $\mathbf{x}^{(0)}$ can be initialized by generating $|V|$ random non-negative numbers and then normalizing them. In the following algorithms, we use $S=\operatorname{RDDOS}(A$, MaxIteration $)$ in Algorithm 1 to represent the procedure of extracting a dominant set $S$ from a weighted graph $G$ given its adjacency matrix $A$ by using replicator dynamics. The stopping criterion of the dynamic system can be set as a maximal iteration number MaxIteration. In this paper, MaxIteration is empirically set to 30 in the experiments. Take the graph shown in Fig. 3 as an example, the matrix $A$ is:

$$
\left(\begin{array}{llll}
0.0 & 0.2 & 0.8 & 0.3 \\
0.2 & 0.0 & 0.3 & 0.7 \\
0.8 & 0.3 & 0.0 & 0.2 \\
0.3 & 0.7 & 0.2 & 0.0
\end{array}\right)
$$

given that $\mathbf{x}^{(0)}$ is initialized as $(0.4,0.3,0.2,0.1)$, after the first iteration of the replicator dynamics defined in Eqn. (6), $\mathbf{x}^{(1)}=(0.35,0.22,0.30,0.13) . \mathbf{x}$ is finally converged to $(0.5,0.0,0.5,0.0)$ after 15 iterations, and we have $x_{1}>0$ and $x_{3}>0$ which indicates that $p_{1}$ and $p_{3}$ form a dominant set, as expected.

\section{Topology estimation via dominant set clustering}

A peeling-off strategy has been proposed in Algorithm 2. which shows the complete procedure of partitioning the points involved in the intersections into branches. It iteratively extracts a subset of points belonging to the same branch (a dominant set $S$ ) each time by using Eqn. (6) and repeating the process with the remaining points in the set $V=V \backslash S$. The identification of different vascular branches at an intersection is carried out by identifying one vascular branch first, and then identifying another from the remaining nodes and segments, and so on. Therefore, the peeling-off strategy is a direct, intuitive implementation of this procedure [14]. As shown in the aforementioned example of Fig. 3 , given that $x^{(0)}$ is initialized as $(0.4,0.3,0.2,0.1)$, the first iteration of Algorithm
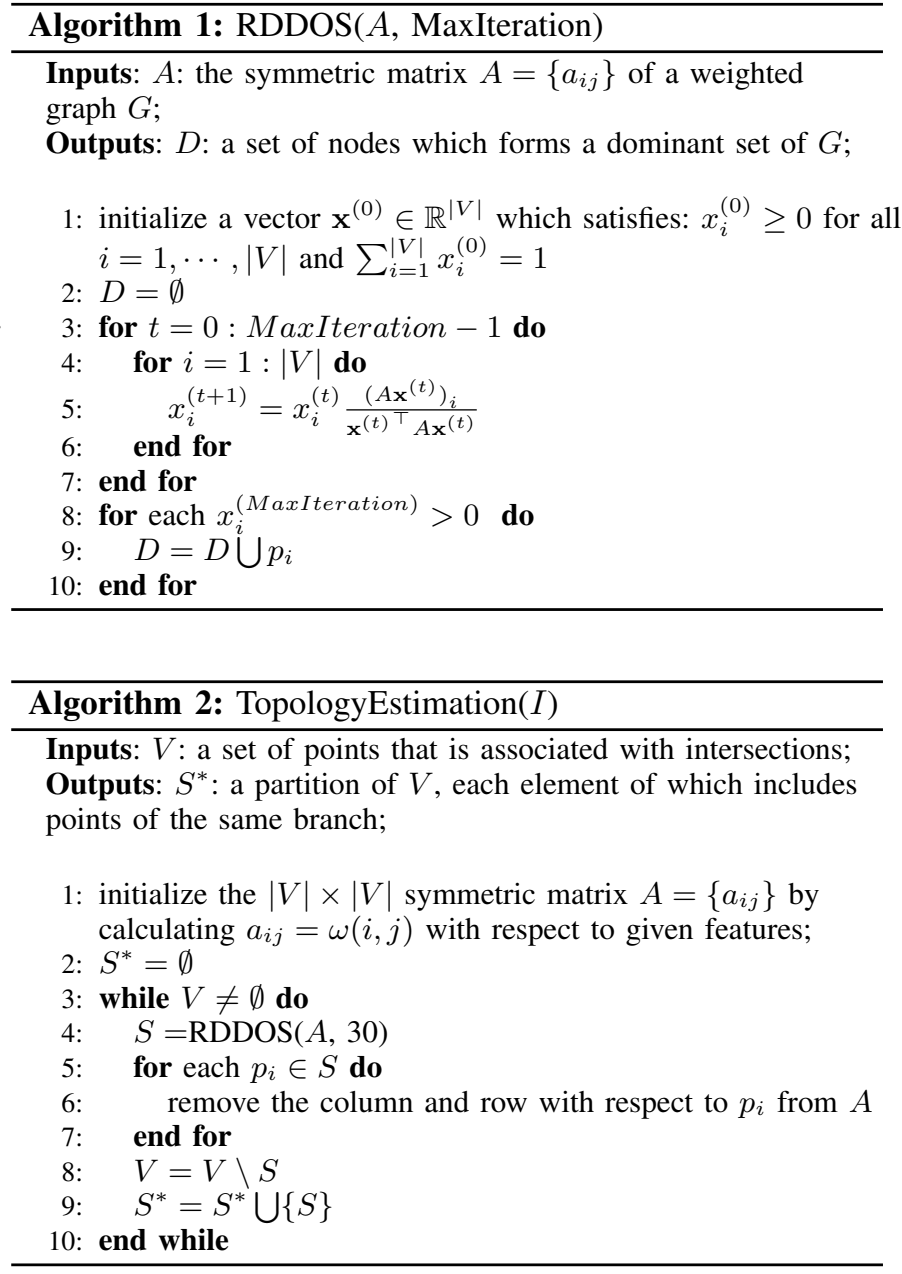

2 identifies the dominant set including $p_{1}$ and $p_{3}$, subsequently the second iteration identifies another including $p_{2}$ and $p_{4}$, as expected.

It is also worth noting that the number of points processed in the process of identifying a dominant set is greatly reduced over different iterations, from the number of intersections in a vascular network after the pre-processing step proposed in Section III to those excluding the vascular branches already identified. This leads to an increase of computational efficiency in the topology estimation.

Each point $p_{i}$ in $V$ is represented as a feature vector $\mathbf{F}_{i}$ : its intensities in $R, G$, and $B$ channels, orientations, curvatures, blood vessel diameters, and entropy. The values of each feature are then linearly normalized individually into the unit interval $[0,1]$. The weight $\omega(i, j)$ of an edge connecting points $p_{i}$ and $p_{j}$ is finally estimated as: $\omega(i, j)=1 /\left(\left\|\mathbf{F}_{i}-\mathbf{F}_{j}\right\|+\epsilon\right)$, where $\|\cdot\|$ denotes the Euclidean distance between $\mathbf{F}_{i}$ and $\mathbf{F}_{j}$. Note that a tiny value $\epsilon=0.000001$ is used in case of $\mathbf{F}_{i}=\mathbf{F}_{j}$.

For each pixel in a vessel segment, 23 features are measured

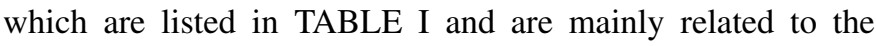
orientation, diameter, and curvature. Most of these features were previously used for topological reconstruction [9], [10] and vessel classification [1], [21] tasks. While other features can be calculated straightforward, the main ones are defined and explained as follows: the orientation of a pixel 
TABLE I: List of features for the representation of points for the proposed DOS classifier.

\begin{tabular}{|l|l|}
\hline No. & Features \\
\hline $1-6$ & $\begin{array}{l}\text { avg. and std. of the intensities within the segment in RGB } \\
\text { channels }\end{array}$ \\
\hline $7-10$ & $\begin{array}{l}\text { avg. and std. of the orientations and curvatures of each } \\
\text { centerline pixels }\end{array}$ \\
\hline $11-12$ & $\begin{array}{l}\text { avg. and std. of the blood vessel diameters of each blood } \\
\text { vessel segment }\end{array}$ \\
\hline $13-18$ & $\begin{array}{l}\text { avg. and std. of the intensities of all centerline pixels under } \\
\text { a Gaussian blurring }(\sigma=4) \text { in RGB channels }\end{array}$ \\
\hline $19-23$ & $\begin{array}{l}\text { entropy of intensity in RGB channels, orientation and curva- } \\
\text { ture of each centerline pixels }\end{array}$ \\
\hline
\end{tabular}

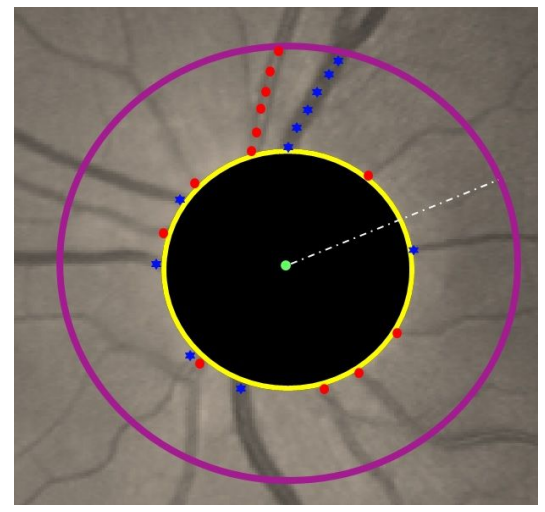

Fig. 4: Definition of a ring for the separation of arteries (red circles) from veins (blue stars).

is defined as the including angle (in radians) between its lying segment and the positive direction of the $x$-axis, a measurement between $[0, \pi][10]$; the diameter of a pixel is measured as a median value of 15 measurements of diametric length between the vessel edges, and passing through the skeleton pixels of the end region [10]; the curvature $C$ of a nerve segment that is represented as an ordered set of pixels $\left(x_{k}, y_{k}\right), k=1,2, \cdots, n$ is computed as $\frac{\Delta x_{k} \Delta^{2} y_{k}-\Delta^{2} x_{k} \Delta y_{k}}{\left[\left(\Delta x_{k}\right)^{2}+\left(\Delta y_{k}\right)^{2}\right]^{3 / 2}}$, where $\Delta$ denotes finite difference, and thus $\Delta x_{k}=x_{k}-x_{k-1}$, $\Delta^{2} x_{k}=\Delta x_{k}-\Delta x_{k-1}, \Delta y_{k}=y_{k}-y_{k-1}$, and $\Delta^{2} y_{k}=$ $\Delta y_{k}-\Delta y_{k-1}$.

We employed the sequential forward floating selection for feature selection method [9], which starts with an empty feature set and adds or removes features when this improves the performance of the classifier. (We refer readers to TABLEI for more details about the selected features.) Fig. 11.e) illustrates the estimated vascular network with the added topological information.

\section{Arteries/Veins classification via dominant set clustering}

As aforementioned, several supervised learning methods have been exploited for the task of $\mathrm{A} / \mathrm{V}$ identification. Their performance is limited due to unavailability of sufficient training data, and complicated structure and training process. In this paper, we take into account the reconstructed vascular topology information, utilize the difference of structural information between arteries and veins reflected in retinal images, and propose the ratio of 'dominant pixels' as a criterion for

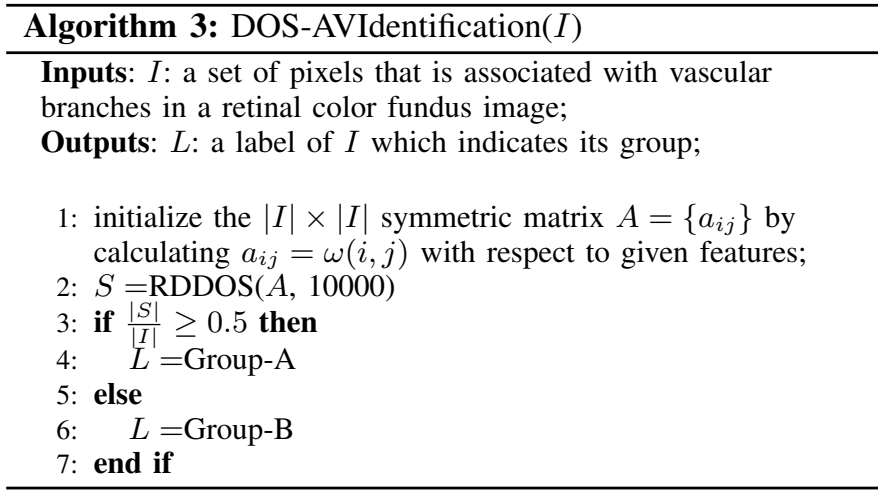

an unsupervised $\mathrm{A} / \mathrm{V}$ identification.

As each branch of retinal blood vessels is composed of many pixels in a retinal image, significant information for discriminating arteries from veins can be derived from their characteristics, and the configuration of the pixels. Dominant pixels are defined as those which form an extracted dominant set amongst all the pixels in a branch of a retinal blood vessel. The dominant pixel ratio is defined as the ratio of the number of dominant pixels to that of all the pixels in a branch of a retinal blood vessel. Based on these definitions, an effective and concise rule is proposed to separate all the branches of blood vessels into two groups: a branch with a high dominant pixel ratio (given 0.5 or greater) is assigned to Group-A, while Group-B includes those branches with a low dominant pixel ratio. Group-A and Group-B are then assigned as either artery or vein based on their intensity and morphology: arteries are brighter in color than veins, and are thinner than neighboring veins [21]. In this work, we define a vessel segment as 'artery' if the average intensity value of the pixels inside the ring centred at any pixel on its centerline with the same radius as the optic disc is larger than 0.48 (with the intensity values lying in the unit interval $[0,1]$ ) (Fig. 4). Otherwise the vessel segment is assigned as 'vein'. The threshold value was chosen empirically. Algorithm 3 shows the details for the identification of Group-A/Group-B pixels via DOS.

The power of the dominant pixel ratio definition for $\mathrm{A} / \mathrm{V}$ identification is derived from the effectiveness of DOS in disclosing the hidden structure in arteries and veins. As with the dominant sets-based vascular topology estimation, the weights assigned to the pixels of a vascular branch is responsible for the quality of $\mathrm{A} / \mathrm{V}$ identification. Once again the features in TABLE [ are used to calculate the weights $\omega(i, j)$. Fig. 1 f f) illustrates the $\mathrm{A} / \mathrm{V}$ classification result.

\section{MATERiAls}

\section{A. Datasets}

Topology reconstruction: Five public retinal datasets were used - the Iowa Normative Set for Processing Images of the REtina (INSPIRE) by the University of Iowa Hospital [1]; the VARPA Images for the Computation of the Arterio/Venular Ratio (VICAVR) captured at the hospital of Conxo, Santiago de Compostela, Spain [3]; the images acquired with 
an ultra-wide-field device (WIDE) by Duke University [5]; the Scanning Laser Ophthalmoscopy technique-based dataset (IOSTAR) from Eindhoven University of Technology [37]; and the well-known Digital Retinal Images for Blood vessel Extraction (DRIVE) dataset [38]. Except the WIDE dataset, the others used for topology estimation do not contain manuallylabeled blood vessel topological information. In consequence, we have annotated them manually, and released these annotation $\mathbb{2}^{2}$ online.

$A / V$ classification: Four of the above mentioned datasets were used: INSPIRE, VICAVR, WIDE, and DRIVE. An image analysis expert manually classified and then an ophthalmologist checked and corrected the blood vessel segments of the INSPIRE, WIDE, and DRIVE datasets into arteries and veins. For the VICAVR dataset, three experts manually labeled the blood vessel segments as arteries or veins, and agreements between them were then used as ground truth. However, the experts only classified the blood vessels found within a radius three times that of the optic disc: in other words, the $\mathrm{A} / \mathrm{V}$ classification labelling for this dataset is incomplete. For a comprehensive visual illustration of $\mathrm{A} / \mathrm{V}$ labels and fair comparison with other datasets, we asked again the image analysis expert to classify and then the ophthalmologist to check and correct all the unlabeled blood vessels as arteries or veins for this dataset.

It is worth noting that the manual labeling for the INSPIRE and WIDE datasets is at blood vessel centerline pixel level, whereas that for the VICAVR, DRVE, and IOSTAR datasets is at blood vessel segment pixel level instead. TABLE II summarizes the important details of all these datasets.

\section{B. Topology ground truth estimation}

As noted above, the DRIVE, INSPIRE, VICAVR, and IOSTAR datasets have no ground truth for blood vessel topology, so two experts were invited to manually label the topological information of the retinal vascular structure by using a graph editing software we developed for the task. Two experts independently labeled each blood vessel segment or centerline for all the datasets, based on the types of available manual annotations or automatic segmentation of the blood vessel structure. The consensus between them was then used as ground truth. Note, we obtained the topology ground truth of DRIVE, INSPIRE, and IOSTAR based on manual segmentations, and for VICAVR, we generated the topology ground truth on automatic segmented vessels by using the method proposed in [34]. For each image, every blood vessel was labeled with a distinct color (or individual label) to clearly distinguish between different blood vessel trees, as shown in Fig. 11(e).

Thus, the blood vessel segments, or blood vessel centerlines used for topology estimation were extracted either by human graders manually or applying an automatic blood vessel segmentation method [34]: i.e., the DRIVE and IOSTAR datasets include the manual annotations of the retinal blood vessels for each image, so the topology reconstruction was made on manual annotated blood vessel patterns; for the

\footnotetext{
${ }^{2}$ http://imed.nimte.ac.cn/vetovessel-topology-groundtruth.html
}

VICAVR dataset, the topology estimation was made at the automatically segmented blood vessels by using the automated segmentation method [34]; for the INSPIRE dataset, the image analysis expert graded and then the ophthalmologist checked and corrected the topology at the blood vessel centerlines, which were generated by the method given in [5].

\section{Evaluation metrics}

To the best of our knowledge, there is no single metric that can measure the performance of a topology estimation procedure, because of the differing properties of multiple scales of the vasculars, i.e., blood vessels of different sizes exhibit properties that cannot be captured by a single metric. Therefore, in this work, we measure the performance of the topology estimation method by calculating the overlap rate of the correctly identified nodes/intersections. Let $A$ be the total number of the nodes of a blood vessel tree, and $B$ be the number of the nodes that have been correctly identified (the vertices of the estimated topology tree have been assigned labels identical to the ground truth). The overlap rate $Q$ is then defined as $Q=\frac{B}{A} \times 100 \%$. Intuitively, $Q$ is the percentage of nodes that are correctly identified by the proposed method [31].

To compare the A/V classification performance of the proposed method with the corresponding ground truth as annotated by our human graders, the following metrics are defined: sensitivity $($ Se $)=T P /(T P+F N) \times 100 \%$, specificity $(S p)=$ $T N /(T N+F P) \times 100 \%$, and balanced accuracy $(B-A c c)=$ $\frac{S e+S p}{2}$, where $T P, T N, F P$ and $F N$ denote true positive, true negative, false positive, and false negative, respectively. For the sake of fair comparison and easy implementation, all the evaluations were undertaken at blood vessel centerline pixel level. In this work, we interpret arteries as positives and veins as negatives. $S e$ shows the ability of a given method to detect arteries, while $S p$ reveals how well it detects veins. $B$ $A c c$ indicates the overall classification performance, and thus reflects the trade-off between sensitivity and specificity [29].

To compare the vessel segmentation performance of different methods, the following metrics are defined: the precision, recall (also known as sensitivity), and false discovery rate (FDR) [39]. Precision quantifies the ratio of the true positive pixels among all the pixels detected as vessel. Recall is the fraction of the number of centerline pixels in the correctly detected segments (true positives) over the total number of the centerline pixels in the ground truth. FDR is defined as the fraction of the total number of the centerline pixels incorrectly detected as vessel segments (false positives) over the total number of the centerline pixels of the traced vessels in the ground truth. The use of specificity, defined as the number of the pixels correctly rejected as non-vessel structures (true negatives), is not adequate for the evaluation of this segmentation task, since the vast majority of the pixels do not belong to blood vessels. It is worth noting that, as is customary in the evaluation of the methods extracting one pixel-wide curves [39], a three-pixel tolerance region around the manually traced nerves is considered to be a true positive. In other words, a predicted centerline point is considered as 
TABLE II: Details of the retinal image datasets used, including the availability of the ground truth for each dataset, and the type of manual annotations.

\begin{tabular}{lcccccc}
\hline \hline Datasets & No. Img. & Size & FOV & Camera & Ground truth & Label Type \\
\hline INSPIRE $^{\dagger}$ & 40 & $2392 \times 2048$ & $30^{\circ}$ & Carl Zeiss Meditec & Topology estimation \& A/V classification & Centerline \\
VICAVR $^{\dagger}$ & 100 & $768 \times 576$ & $45^{\circ}$ & Cannon CR6-45NM & Topology estimation \& A/V classification & Segment \\
DRIVE $^{\dagger}$ & 40 & $565 \times 584$ & $45^{\circ}$ & Cannon CR5-NM3-CCD & Topology estimation \& A/V classification & Segment \\
WIDE $^{\dagger}$ & 30 & $1440 \times 900$ & $45^{\circ}$ & Optos 200Tx & Topology estimation \& A/V classification & Centerline \\
IOSTAR $^{\dagger}$ & 30 & $1024 \times 1024$ & $45^{\circ}$ & EasyScan & Topology estimation & Segment \\
\hline
\end{tabular}

true positive if there are no more than three pixels from the nearest ground truth centerline point.

\section{EXPERIMENTAL RESUlTS}

In order to validate the effectiveness of the proposed DOS algorithm for retinal vascular structure analysis, we have thoroughly evaluated it over five publicly accessible datasets as described in the last section.

\section{A. Topology estimation}

Fig. 5 illustrates five vascular topology reconstruction results, each derived from one of these retinal datasets: INSPIRE, IOSTAR, VICAVR, DRIVE, and WIDE, respectively. Compared with the manual annotations, as shown in the second column of Fig. 5, it is clear from visual inspection that our method is able to identify most nodes correctly: only a few cases were incorrectly traced, and these were located at crossovers, as shown in the last column of Fig. 5- the red squares indicate incorrectly-traced significant nodes.

To better facilitate observation and objective evaluation of the performance of the proposed method in the reconstruction of blood vessel topology, the overlap rates $Q$ of the relevant significant nodes were also calculated. TABLE III presents the performance measurements of the proposed method in identifying connectivity of these nodes at bifurcations (BIF) only, crossovers (CRO) only, and all nodes, by counting the number of true positives (TP: correctly identified nodes). As expected, the $Q$ scores for bifurcation points for all the five datasets are much higher than those at crossovers, since it is a relatively simpler node analysis task. The average overlap score over both bifurcations and crossovers was also calculated for all the datasets, and it reveals that the proposed method has the best performance on the INSPIRE dataset with an average $Q=95.1 \%$, while yielding the lowest score on the DRIVE dataset, with an average $Q=91.1 \%$. This is due to the relatively low resolution and image contrast of this dataset.

In addition, the accuracy of the topology reconstruction at blood vessel centerline level was calculated, and it can be observed that the accuracy scores are higher than the corresponding average overlap rate: for example, we see an accuracy score of $97.5 \%$, but an average overlap rate of only $95.1 \%$ for the INSPIRE dataset. This is because the number of blood vessel segments is much larger than the number of significant nodes (bifurcation and crossovers).

As we mentioned above, there is no single metric that is able to directly and objectively measure the performance of a topology reconstruction method. The most common method
TABLE III: Performance of the proposed method on topology reconstruction at node and segment level, respectively, over different datasets.

\begin{tabular}{c|ccccc}
\hline \hline & INSPIRE & IOSTAR & VICAVR & DRIVE & WIDE \\
\hline \# BIF & 1998 & 1213 & 4955 & 2478 & 3678 \\
\# TP & 1945 & 1167 & 4799 & 2288 & 3551 \\
$\boldsymbol{Q}$ score & $97.3 \%$ & $96.2 \%$ & $96.8 \%$ & $92.3 \%$ & $96.5 \%$ \\
\hline \# CRO & 778 & 482 & 1421 & 832 & 1230 \\
\# TP & 697 & 431 & 1182 & 728 & 1107 \\
$\boldsymbol{Q}$ score & $89.7 \%$ & $89.4 \%$ & $83.2 \%$ & $87.5 \%$ & $90.0 \%$ \\
\hline ave. $\boldsymbol{Q}$ & $95.1 \%$ & $94.2 \%$ & $93.8 \%$ & $91.1 \%$ & $94.9 \%$ \\
\hline Accuracy & $97.5 \%$ & $95.7 \%$ & $94.6 \%$ & $93.5 \%$ & $96.4 \%$ \\
\hline
\end{tabular}

as described in [5], [31] was topological structure-guided A/V classification. Therefore, in the following subsection, we continue to make use of the proposed DOS-based topology estimation method to guide the task of $\mathrm{A} / \mathrm{V}$ classification.

\section{B. A/V classification}

After the reconstruction of the blood vessel topology, the complete blood vessel network has been separated into several individual branches as sub-trees with an individual label (distinct color). The final goal is to assign these labels to one of two classes: artery or vein.

The features listed in TABLE I and the DOS classifier were utilized again to classify these individual labels into two clusters, $A$ and $B$. For each sub-tree $v$, the probability of its being $A$ is estimated by the number of blood vessel pixels classified by DOS as $A$ : $P_{A}^{v}=n_{A}^{v} /\left(n_{A}^{v}+n_{B}^{v}\right)$, where $n_{A}^{v}$ is the number of pixels classified as $A$, and $n_{B}^{v}$ is the number of pixels classified as $B$. For each sub-tree, the higher probability is used to define whether the sub-tree is assignable to category $A$ or $B$. Clusters $A$ and $B$ are then assigned as artery and vein, respectively, based on their intensity and morphology: arteries are brighter in color than veins, and are thinner than neighboring veins [21].

Fig. 6 shows the $\mathrm{A} / \mathrm{V}$ classification results of the proposed method over four sample images, one from each dataset, based on their topological information. Overall, the proposed method can correctly assign most of the A/V labels on all the four datasets, in comparison with the corresponding manual annotations. The arteries are shown in red and veins in blue. However, our $\mathrm{A} / \mathrm{V}$ classification relies heavily on the prior results of topology reconstruction: if a blood vessel segment was incorrectly identified at the stage of topology reconstruction, it might be very likely to be falsely labeled during subsequent $\mathrm{A} / \mathrm{V}$ classification. This is because a blood 


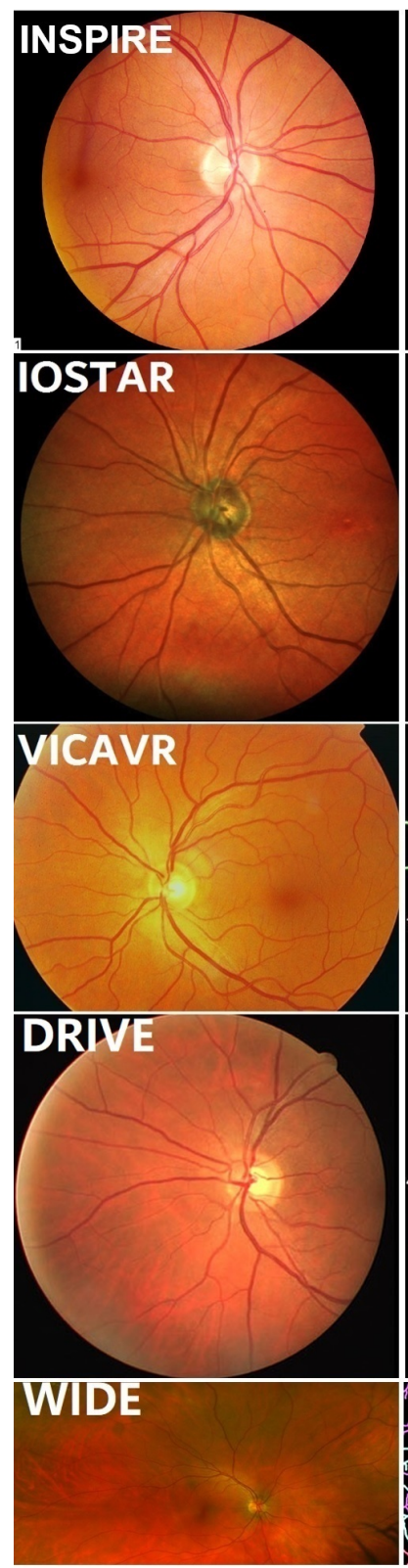

Original image
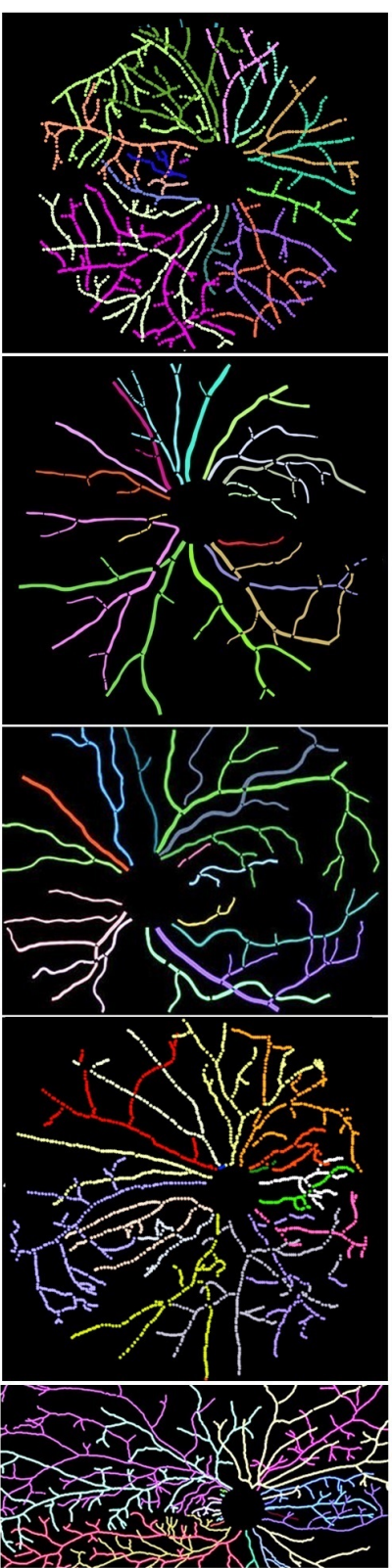

Ground truth
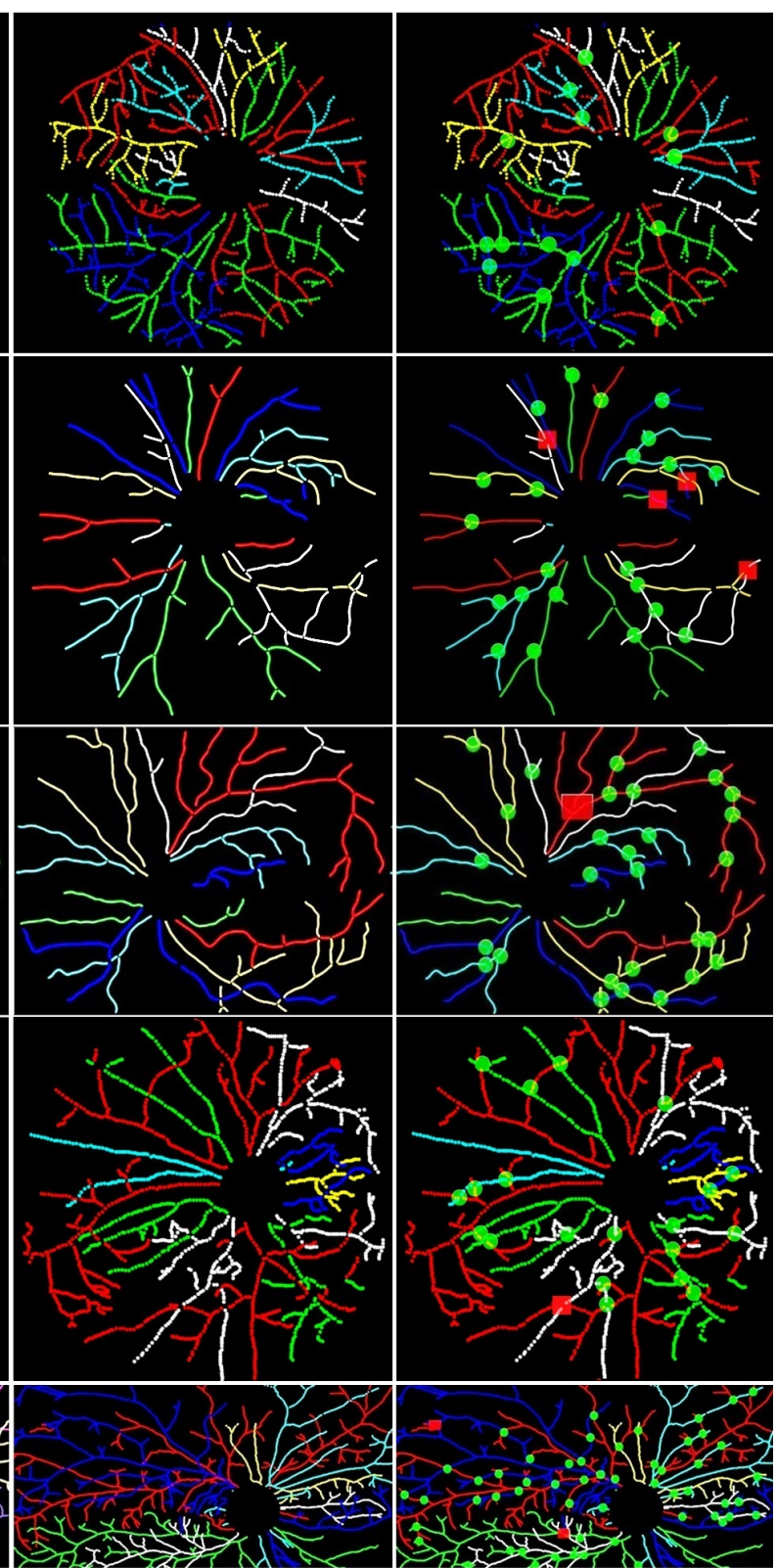

Estimated Topology

Fig. 5: Examples of vascular topology estimation results. From left to right column: original image, manual annotation, result of the proposed method, and the highlighted correctly (Green discs) and incorrectly (Red squares) identified connections.

vessel segment must share its label with all its downstream segments, as evidenced by the figures in the second and third columns of Fig. 6 - the incorrectly traced veins are labeled in green, while the falsely identified arteries are labeled in yellow.

In order to better demonstrate the superiority of the proposed method, TABLE IV reports the comparison of our method with the state-of-the-art A/V ones over four datasets, in terms of centerline pixel-level sensitivity, specificity, and balanced accuracy. It is shown that our method correctly identifies over $93.5 \%$ of the A/V labels of all the datasets, outperforming all the compared methods, with a single exception that its $S p$ score on the DRIVE dataset is $1.4 \%$ lower than that of [29]. Nevertheless, the proposed method is able to correctly identify the majority of arteries and veins by relying on the topological constraint.

The intra-observer scores are also provided for the INSPIRE and WIDE datasets in TABLE IV] It can be seen that the sensitivities of the proposed method are very close to those of the human observers: our method obtains competing sensitivities, with $96.8 \%$ and $96.2 \%$, compared to $97.2 \%$ and $97.0 \%$ by the intra-observers for the INSPIRE and WIDE datasets, respectively.

Overall, the proposed method has the best performance on the INSPIRE dataset, with a balanced accuracy of $96.4 \%$. This dataset contains images with a higher resolution, and considerably fewer and simpler blood vessel bifurcations and crossovers (e.g., an average number 40 vs. 102 of circuits 

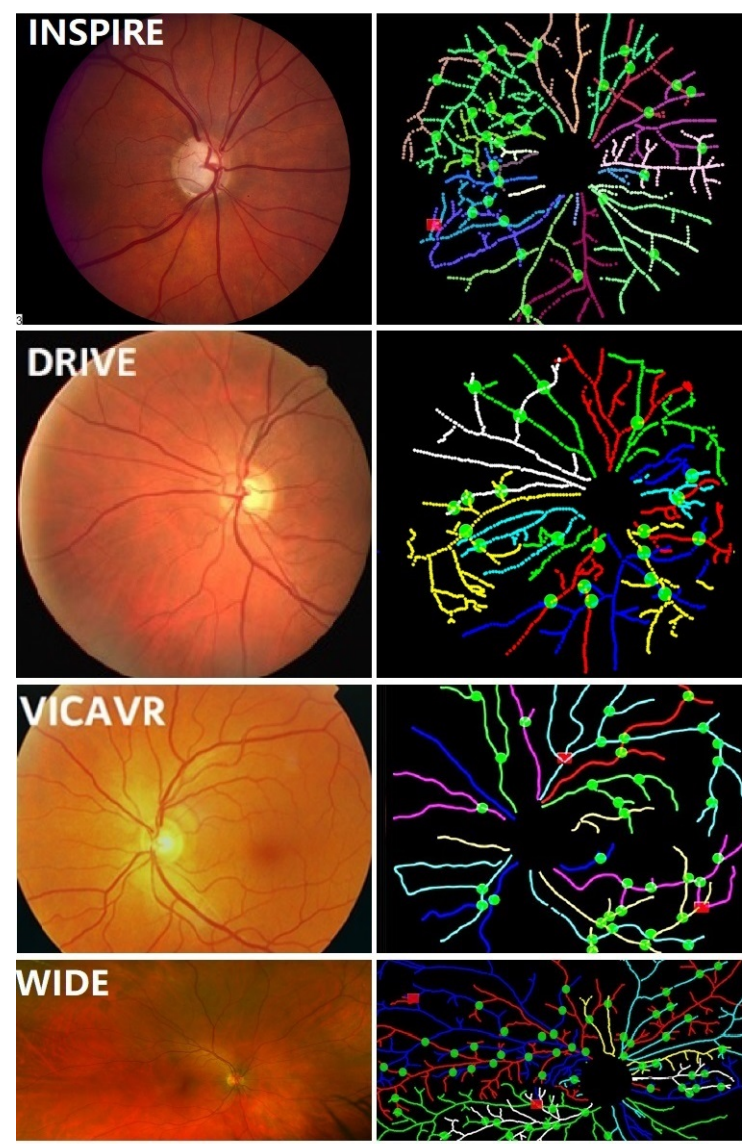

Original image
Estimated topology

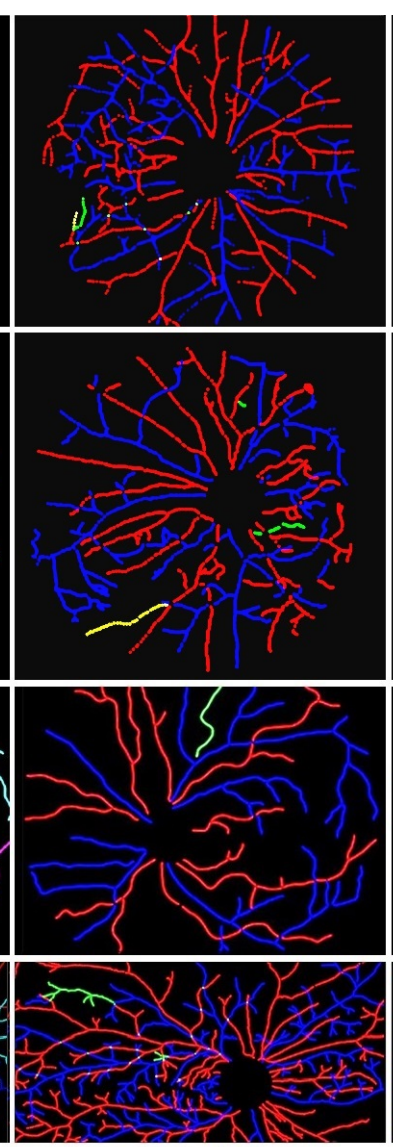

Automated result
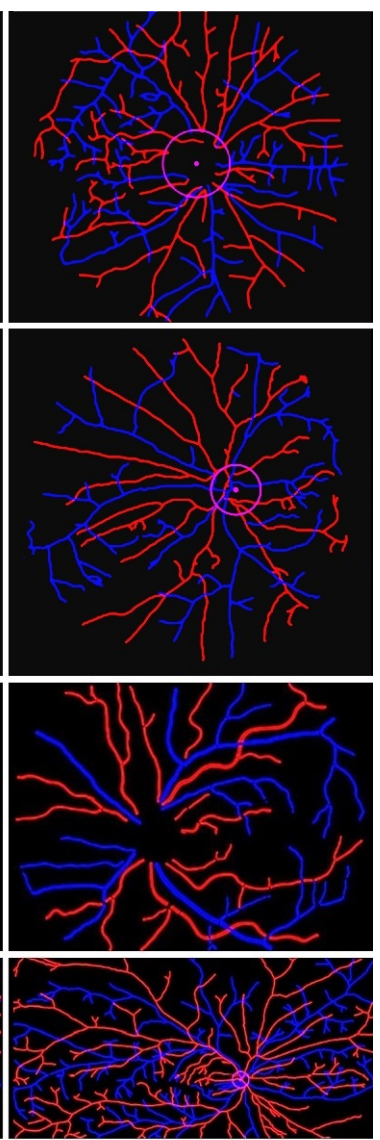

Ground truth

Fig. 6: A/V classification results of the proposed method over four different datasets. From left to right column: original image, blood vessel topology, A/V classification result, and corresponding manual annotations.

when compared to the WIDE dataset). This finding is also evidenced by the performance on the topology estimation, with the highest accuracy of $97.5 \%$, when compared with other datasets in TABLE III

All the above-reported performances of different methods over the DRVIE dataset were obtained when using the manual annotation produced by Qureshi et al. [42] as ground truth. It is noted that the original authors of the DRIVE dataset also released another manual $\mathrm{A} / \mathrm{V}$ annotation [38]. We refer them as GT-1 and GT-2, respectively. Fig. 7 shows two examples of these two manual $\mathrm{A} / \mathrm{V}$ annotations.

In order to demonstrate our A/V classification method over two different manual annotations, and the agreement of those manual annotations, we computed the false discovery rate (FDR) [39] in three different scenarios: Auto vs GT-1, Auto vs GT-2, and GT-1 vs GT-2. It can be observed from TABLE $\mathrm{V}$ that the proposed automatic A/V classification method has a larger FDR than the human annotations, as expected, and the two human annotations also have an FDR of as large as $0.071 \pm 0.014$. Interestingly, Estrada et al. [29] also reported a very close agreement score of $96 \%$ between GT-1 and GT-2. These differences between the annotations given by different human graders imply the difficulty of the $\mathrm{A} / \mathrm{V}$ classification problem.

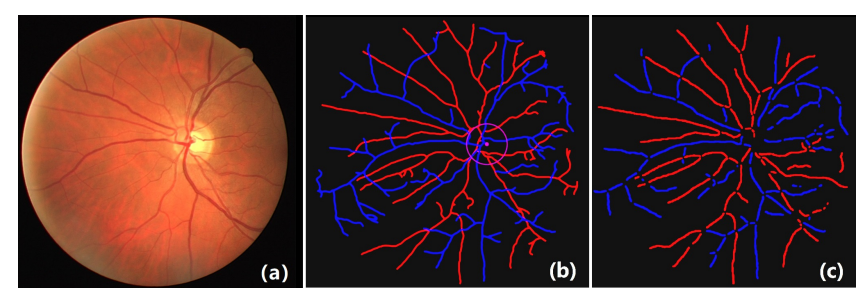

Fig. 7: Different manual A/V annotation results of the DRIVE dataset. (a) original image, (b) manual annotation by [42] (GT-1), (c) manual annotation by [38] (GT-2).

TABLE V: False discovery rate of different A/V classification methods over two different manual annotations: GT-1 and GT2. Auto refers to the proposed automated $\mathrm{A} / \mathrm{V}$ classification method.

\begin{tabular}{c|ccc}
\hline & Auto $v s$ GT-1 & Auto $v$ GT-2 & GT-1 vs GT-2 \\
\hline FDR & $0.093 \pm 0.009$ & $0.088 \pm 0.013$ & $0.071 \pm 0.014$ \\
\hline
\end{tabular}

\section{Computational complexity}

The proposed method has a computational complexity of $O\left(N^{2}\right)$ in topology estimation, where $N$ is the total number of nodes in a blood vessel graph, a computational complexity of $O\left(M \times P^{2}\right)$ in label assignment of each vessel branch, 
TABLE IV: The sensitivity $S e$, specificity $S p$ and balanced accuracy $B$-Acc of different A/V classification methods over different datasets.

\begin{tabular}{|c|c|c|c|c|c|c|c|c|c|c|c|c|c|}
\hline \multirow{2}{*}{ (n) } & \multirow[b]{2}{*}{ Classifier } & \multicolumn{3}{|c|}{ INSPIRE } & \multicolumn{3}{|c|}{ DRIVE } & \multicolumn{3}{|c|}{ VICAVR } & \multicolumn{3}{|c|}{ WIDE } \\
\hline & & $\mathbf{S e}$ & Sp & B-Acc & Se & Sp & B-Acc & Se & Sp & B-Acc & Se & Sp & B-Acc \\
\hline Second human grader & - & $97.2 \%$ & $97.0 \%$ & $97.1 \%$ & - & - & - & - & - & - & $97.0 \%$ & $97.9 \%$ & $97.4 \%$ \\
\hline Niemeijer et al. $[\overline{1}]$ & kNN & $78.0 \%$ & $78.0 \%$ & $78.0 \%$ & $80.0 \%$ & $80.0 \%$ & $80.0 \%$ & - & - & - & - & - & - \\
\hline Muramatsu et al. 140 & LDA & - & - & - & - & - & $93.0 \%$ & - & - & - & - & - & - \\
\hline Vazquez et al. $|\overline{3}|$ & k-means & - & - & - & - & - & - & - & - & $88.8 \%$ & - & - & - \\
\hline Mirsharif et al. 233 & LDA & - & - & - & - & - & $84.1 \%$ & - & - & - & - & - & - \\
\hline Relan et al. $|24|$ & GMM & $92.7 \%$ & $48.5 \%$ & $70.8 \%$ & - & - & - & - & - & - & - & - & - \\
\hline Dashbozorg et al. [9] & LDA & $91.0 \%$ & $86.0 \%$ & $88.5 \%$ & $90.0 \%$ & $84.0 \%$ & $87.0 \%$ & - & - & $89.8 \%$ & - & - & - \\
\hline Lyu et al. $[30]$ & k-means & $90.2 \%$ & $79.4 \%$ & $84.8 \%$ & - & - & - & - & - & - & - & - & - \\
\hline Pellegrini et al. $[41]$ & graph & - & - & - & - & - & & - & - & - & - & - & $92.5 \%$ \\
\hline Girard et al. 25 & CNN & - & - & - & $92.3 \%$ & $93.1 \%$ & $92.7 \%$ & - & - & - & - & - & - \\
\hline Huang et al. $[\overline{26} \mid$ & LDA & - & - & $85.1 \%$ & - & - & - & - & - & $90.6 \%$ & - & - & - \\
\hline Estrada et al. 29 & graph & $\begin{array}{l}91.5 \% \\
\pm 0.1 \%\end{array}$ & $\begin{array}{l}90.2 \% \\
\pm 0.1 \%\end{array}$ & $\begin{array}{l}90.9 \% \\
\pm 0.1 \%\end{array}$ & $\begin{array}{l}93.0 \% \\
\pm 0.06 \%\end{array}$ & $\begin{array}{l}\mathbf{9 4 . 1 \%} \\
\pm 0.07 \%\end{array}$ & $\begin{array}{l}93.5 \% \\
\pm 0.05 \%\end{array}$ & - & - & - & $\begin{array}{l}91.0 \% \\
\pm 0.06 \%\end{array}$ & $\begin{array}{l}90.9 \% \\
\pm 0.06 \%\end{array}$ & $\begin{array}{l}91.0 \% \\
\pm 0.06 \%\end{array}$ \\
\hline Proposed & DOS & $\begin{array}{l}\mathbf{9 6 . 8 \%} \\
\pm 0.08 \%\end{array}$ & $\begin{array}{l}\mathbf{9 5 . 7 \%} \\
\pm 0.08 \%\end{array}$ & $\begin{array}{l}\mathbf{9 6 . 4 \%} \\
\pm 0.06 \%\end{array}$ & $\begin{array}{l}\mathbf{9 4 . 2 \%} \\
\pm 0.07 \%\end{array}$ & $\begin{array}{l}92.7 \% \\
\pm 0.07 \%\end{array}$ & $\begin{array}{l}\mathbf{9 3 . 5 \%} \\
\pm 0.07 \%\end{array}$ & $\begin{array}{l}\mathbf{9 5 . 4 \%} \\
\pm 0.1 \%\end{array}$ & $\begin{array}{l}\mathbf{9 3 . 8 \%} \\
\pm 0.09 \%\end{array}$ & $\begin{array}{l}\mathbf{9 4 . 6 \%} \\
\pm 0.09 \%\end{array}$ & $\begin{array}{l}\mathbf{9 6 . 2 \%} \\
\pm 0.1 \%\end{array}$ & $\begin{array}{l}\mathbf{9 4 . 2 \%} \\
\pm 0.06 \%\end{array}$ & $\begin{array}{l}\mathbf{9 5 . 2 \%} \\
\pm 0.08 \%\end{array}$ \\
\hline
\end{tabular}

where $M$ is the total number of individual vessel branches in a vessel graph, and $P$ is the average number of pixels in an individual vessel branch and a computational complexity of $O(M)$ in identifying the labels of the vessel branches as either artery or vein. Therefore, the entire computational complexity of the proposed method is $O\left(N^{2}\right)+O\left(M \times P^{2}\right)$ and thus $O\left(N^{2}\right)$ where $M \leq N, P \leq N$, and $M P \leq N$.

The average running times of our method with standard deviation in seconds for overall $\mathrm{A} / \mathrm{V}$ classification and topology estimation are: $72.61 \pm 16.32 \mathrm{~s}$ and $2.83 \pm 0.41 \mathrm{~s}$ over INSPIRE; $24.43 \pm 2.31 \mathrm{~s}$ and $2.10 \pm 0.33 \mathrm{~s}$ over DRIVE; $28.98 \pm 0.48 \mathrm{~s}$ and $2.57 \pm 1.01 \mathrm{~s}$ over VICAVR; and $33.77 \pm 2.01 \mathrm{~s}$ and $5.84 \pm 1.37 \mathrm{~s}$ over WIDE respectively. Note, we did not perform the A/V classification over IOSTAR due to lack of manual annotation, so we only report the running time of its topology estimation as $1.93 \pm 0.80 \mathrm{~s}$. All the experiments were carried out in MATLAB2015a on a PC with an Intel Xeon E5-2695 v4 CPU, 2.10GHz, and 128GB RAM.

In the literature, only the method proposed by Estrada et al. [29] reported the computation time of their A/V classification on a Toshiba Satellite X870 laptop with a $2.4 \mathrm{Ghz}$ Intel I7 quad-core processor and 32GB RAM: 117.68 \pm 34.10 s over INSPIRE; $131.32 \pm 33.40$ s over DRIVE; and $777.35 \pm 330.52 \mathrm{~s}$ over WIDE.

\section{DisCUSSIONS AND CONCLUSIONS}

In this work, we proposed a DOS-based topology estimation method, and further utilized the obtained topological information to separate arteries from veins in retinal fundus images. In other words, the blood vessel classification problem has been formalized as a pairwise clustering problem. In this section, we carry out a comprehensive comparison between the proposed method and the state-of-the-art label propagation (or topology estimation) and $\mathrm{A} / \mathrm{V}$ classification methods.

\section{A. Comparison to the state-of-the-art label propagation / topology estimation methods}

As suggested by De et al. [32], the topology estimation task can be reformulated as topological label propagation over
TABLE VI: The overlapping rate ( $Q$ score) of different label propagation and topology estimation methods over different datasets.

\begin{tabular}{l|ccccc}
\hline \hline & INSPIRE & IOSTAR & VICAVR & DRIVE & WIDE \\
\hline LBP [43 & $76.6 \%$ & $75.9 \%$ & $74.6 \%$ & $71.9 \%$ & $72.3 \%$ \\
ZFL [45] & $84.3 \%$ & $84.3 \%$ & $83.1 \%$ & $82.7 \%$ & $81.7 \%$ \\
SGL [44] & $81.0 \%$ & $79.8 \%$ & $82.0 \%$ & $80.9 \%$ & $78.8 \%$ \\
MFTD [31] & $91.1 \%$ & $90.9 \%$ & $87.6 \%$ & $84.9 \%$ & $87.5 \%$ \\
HSA [5] & - & - & - & - & $89.6 \%$ \\
\hline DOS & $\mathbf{9 5 . 1 \%}$ & $\mathbf{9 4 . 2 \%}$ & $\mathbf{9 3 . 8 \%}$ & $\mathbf{9 1 . 1 \%}$ & $\mathbf{9 4 . 9 \%}$ \\
\hline
\end{tabular}

the directed graph. Therefore, in this section, the $Q$ values at blood vessel centerline pixel level obtained by the proposed DOS method is compared with those of five state-of-the-art label propagation and topology estimation methods: Loopy Belief Propagation (LBP) [43], Symmetrized Graph Laplacian (SGL) [44], Zero-mode Free Laplacian (ZFL) [45], MatrixForest Theorem of Directed graphs (MFTD) [31], [46], and Heuristic Search Algorithm (HSA) [5]. Of these methods, LBP, SGL, and ZFL are essentially label propagation approaches, for which the source codes have already been developed by the machine learning community; MFTD and HSA are state-of-the-art topology reconstruction methods for tree-like structures: their codes are available from the authors. However, the HSA was designed for the WIDE dataset, and is thus applicable only to this dataset.

In our experiments, the recommended parameters from the original source code or literature were used. These methods all share the same blood vessel extraction and optic disc removal step, as well as the preparation process of converting the skeleton maps into undirected graphs or digraphs, as described in Section III.A. The experimental results are presented in TABLE VI.

Overall, our DOS method consistently outperforms the other label propagation methods [43]-[45] and existing topology estimation methods [5], [31] by a rather large margin. Again, most methods yielded their best results over the INSPIRE dataset due to its simpler branches.

Fig. 8 also presents for visual comparison the topology reconstruction results of the competing methods on an example 


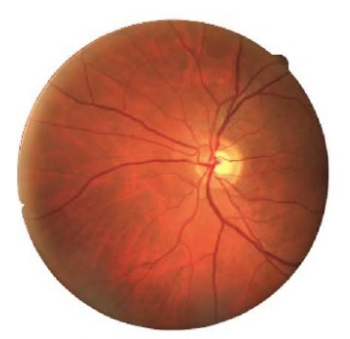

Original image

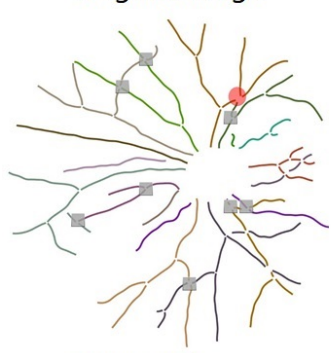

ZFL result

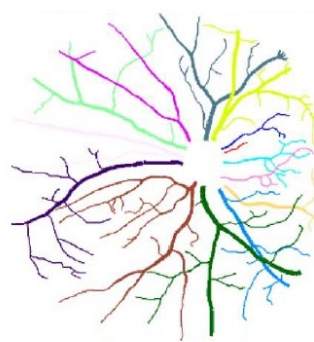

Groundtruth

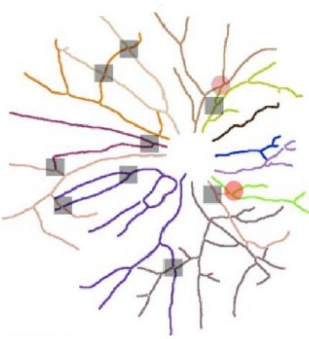

MFTD result

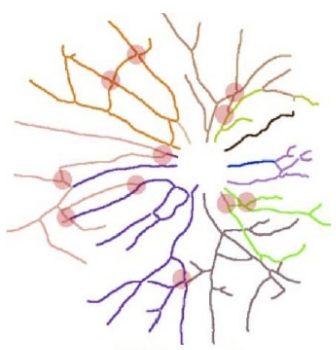

LBP result

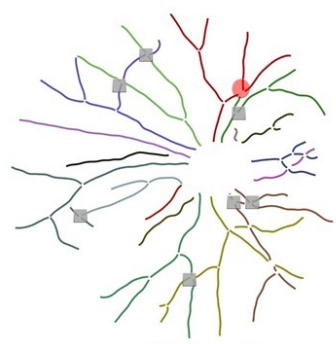

HSA result

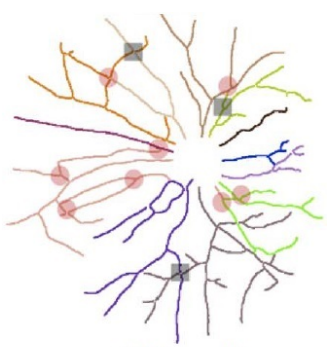

SGL result

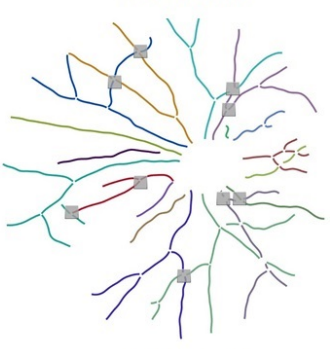

DOSresult

Correct

Fig. 8: The results of different methods in determining the labels at bifurcations and crossovers. Note: the results of LBP, SGL, and MFTD were quoted from [31] for convenience.
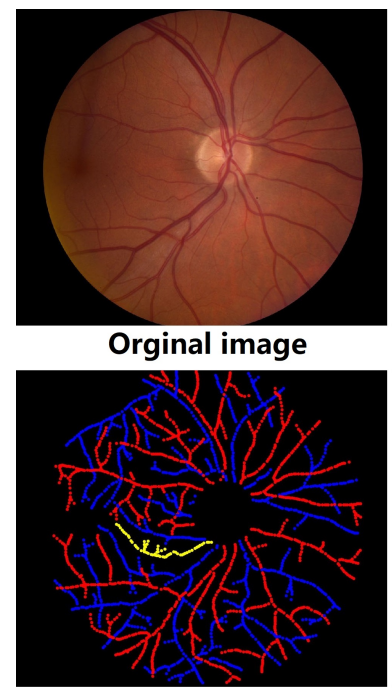

SVM, B-ACc $=93.4 \%$

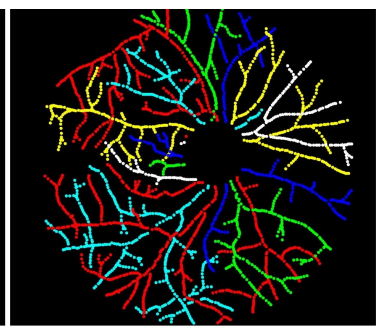

Estimated topology

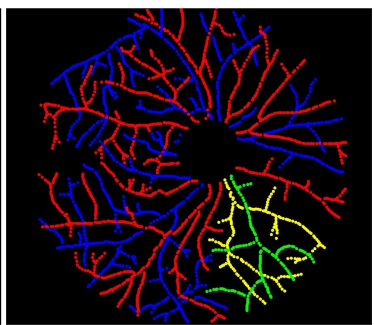

k-means, B-Acc $=\mathbf{8 3 . 5} \%$

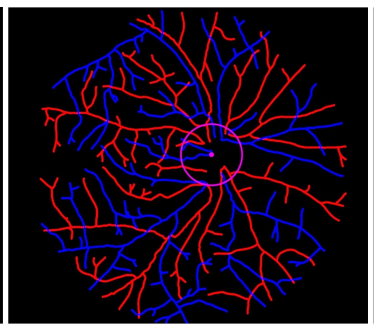

Groundtruth

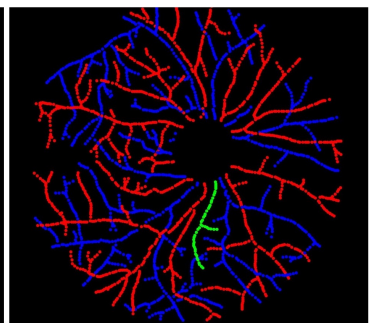

GMM, B-Acc $=93.1 \%$

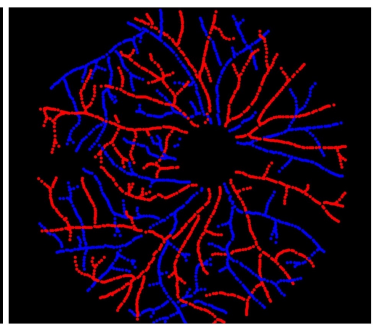

DOS, B-Acc $=100 \%$

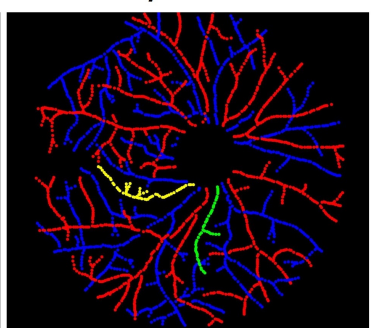

LDA, B-ACC $=88.1 \%$

Fig. 9: Blood vessel classification results of a randomly selected image from the INSPIRE dataset using different A/V classifiers over the topology-assigned blood vessel structures produced by the proposed method.

image from the DRIVE dataset. Grey and red colored disks are used here to represent correct and incorrect predictions in various bifurcation and crossover scenarios. It can be seen that the proposed method produces better topology reconstruction results than its competitors, by observing the bifurcations and crossovers where these have been highlighted. This is because MFTD takes into consideration only the angular or intensity properties of neighbouring blood vessel segments forming a junction for the construction of their current weight matrix [31]. Generally speaking, the proposed method achieves the best performance, which suggests the advantages of our DOS approach for vascular topology reconstruction. However, the proposed method also makes errors in some cases, since it may suffer from failures at the segmentation and skeletonization stages that may misrepresent the topological structures of the retinal blood vessels for the classification phase.

\section{B. Comparison to the state-of-the-art $A / V$ classifiers}

To facilitate better comparison with the results of our classifier, we also applied the most commonly-used A/V classifiers to the topology-assigned structures derived by our method. The comparative A/V classifiers chosen were LDA, GMM, k-means clustering, and SVM. For the supervised classifiers (LDA and SVM), we randomly assigned half of the images as a training set, and the remainder of the images as the test set. The same features listed in TABLE \ were used to 
TABLE VII: The balanced accuracy and standard deviation of different classifiers over the topology-assigned structure derived by the proposed method from different datasets.

\begin{tabular}{l|cccc}
\hline \hline & INSPIRE & DRIVE & VICAVR & WIDE \\
\hline LDA & $91.6 \% \pm 0.10 \%$ & $89.6 \% \pm 0.11 \%$ & $91.1 \% \pm 0.10 \%$ & $90.6 \% \pm 0.12 \%$ \\
GMM & $92.1 \% \pm 0.09 \%$ & $87.4 \% \pm 0.06 \%$ & $91.0 \% \pm 0.07 \%$ & $89.3 \% \pm 0.08 \%$ \\
k-means & $88.3 \% \pm 0.12 \%$ & $85.8 \% \pm 0.11 \%$ & $90.5 \% \pm 0.10 \%$ & $90.1 \% \pm 0.12 \%$ \\
SVM & $93.9 \% \pm 0.07 \%$ & $88.3 \% \pm 0.09 \%$ & $90.6 \% \pm 0.06 \%$ & $89.2 \% \pm 0.08 \%$ \\
\hline DOS & $\mathbf{9 6 . 4 \%} \pm 0.06 \%$ & $\mathbf{9 3 . 5 \%} \pm 0.07 \%$ & $\mathbf{9 4 . 6 \%} \pm 0.09 \%$ & $\mathbf{9 5 . 2 \%} \pm 0.08 \%$ \\
\hline
\end{tabular}

train the LDA and SVM classifiers. TABLE VII demonstrates how well the competing classifiers succeeded in classifying the topology-assigned network into arteries or veins. It can be seen that our DOS method clearly outperformed the compared classification methods, with a balanced accuracy $(B-A c c)$ of $96.4 \%, 93.5 \%, 94.6 \%$, and $95.2 \%$ on the INSPIRE, DRIVE, VICAVR, and WIDE datasets, respectively.

Fig. 9 illustrates the $\mathrm{A} / \mathrm{V}$ classification results of different classifiers on our DOS-guided blood vessel topology (Green: incorrectly traced veins; Yellow: incorrectly traced arteries). It shows that the proposed DOS method achieved the highest balanced accuracy in this case, with $100 \%$ on a randomly-selected image from the INSPIRE dataset. The k-means clustering classifier produced the worst results with a $B-A c c$ score of 83.5\%: this is because one of its major drawbacks is the naive use of the mean value for the representation of each cluster, as a result of which the clusters obtained may not be repeatable and lack consistency. The GMM method obtained relatively higher scores than the other three compared methods, with a $B$ Acc score of $93.1 \%$ : it is more flexible in terms of cluster shape representation than k-means and SVM, and the clusters can be shaped as ellipses, rather than being restricted to hyperspheres in k-means clustering or hyperplanes in SVM.

From a comparison of TABLE[IV] and TABLE[VII it can be observed that the method proposed by Dashtbozorg et al. [9] yielded a $B$-Acc score of $88.5 \%$ over the INSPIRE dataset: they used similar features to train the LDA classifier. After the LDA classifier was applied to our DOS generated blood vessel topology, the $B-A c c$ score has been dramatically increased to 91.6\%. In a similar manner to the GMM classifier, the $B-A c c$ score has been increased from $70.8 \%$ [24] to $92.1 \%$. These results imply that the accurate identification of the underlying network topology is key to improving the accuracy of $\mathrm{A} / \mathrm{V}$ classification.

\section{Feature importance analysis}

The feature weights for nodes and blood vessel segments are critical to the accuracy of subsequent topology estimation and $\mathrm{A} / \mathrm{V}$ classification. In real applications, the feature weights are usually determined by a similarity measure, where only certain features are taken into consideration. Many strategies have been explored for the selection of these critical features, in an effort to identify more compact and better quality feature subsets for various tasks. Such techniques typically involve the use of an individual feature significance evaluation, or a measurement of feature subset consistency, that work together with a search algorithm to determine a quality subset of features. However, most of the feature selection techniques are supervised, i.e., the labels of instances are required for evaluating or measuring the significance of the feature subsets. For the purposes of this topology estimation and $\mathrm{A} / \mathrm{V}$ classification work, it is prohibitively time-consuming to have the vascular branches annotated by human experts, and hence benchmarking public data sets are rare in the literature. Therefore, the features used here to determine the weights $(\omega(i, j)$ in Algorithm 3$)$ were empirically selected, as listed in TABLE I]

We reported the performances of topology estimation and A/V classification results of the proposed method in TABLE VIII in terms of removing the intensity-based, orientationbased, and diameter-based features, respectively from the feature candidates. It can be seen that intensity-based features affect most of the proposed classifier, as the $Q$ scores of the topology estimation are significantly decreased by $4.1 \%, 6.0 \%$, $5.5 \%, 3.0 \%$, and $2.9 \%$ over INSPIRE, DRIVE, VICAVR, WIDE, and IOSTAR, respectively after their removal. Similarly, the $B-A c c$ scores of $\mathrm{A} / \mathrm{V}$ classification are also decreased by a significant margin: $4.3 \%, 5.0 \%, 4.0 \%$, and $3.1 \%$ over INSPIRE, DRIVE, VICAVR, and WIDE datasets.

Fig. 10 illustrates the resulting topology estimation and A/V classification labels of a randomly selected image from the VICAVR database (Green: incorrectly traced veins; Yellow: incorrectly traced arteries), after removing the intensity-based, orientation-based, and diameter-based features individually from the feature representation of the pixels of interest. It demonstrates that intensity-based features are the most important: more incorrectly identified connections occurred, and more incorrectly classified arteries and veins took place after their removal.

\section{Comparison between automatic and manual segmentations}

All the above-reported results are based on ground truth segmentation, with a single exception for the VICAVR dataset. In this subsection, in order to characterize how the errors of automatic and manual segmentations affect their topological reconstructions and final $\mathrm{A} / \mathrm{V}$ classifications, we have repeated our topology estimation and $\mathrm{A} / \mathrm{V}$ classification methods over the automated vessel segmentation results of the INSPIRE dataset.

To compare the vessel segmentation performance of the automated method with the corresponding ground truth, we computed the precision, recall (also known as sensitivity), and false discovery rate (FDR) [39] between the predicted centerlines and ground truth centerlines (the INSPIRE dataset provides the centerline of the retinal blood vessels only). The experimental results are presented in Figure 11 and Table IX 


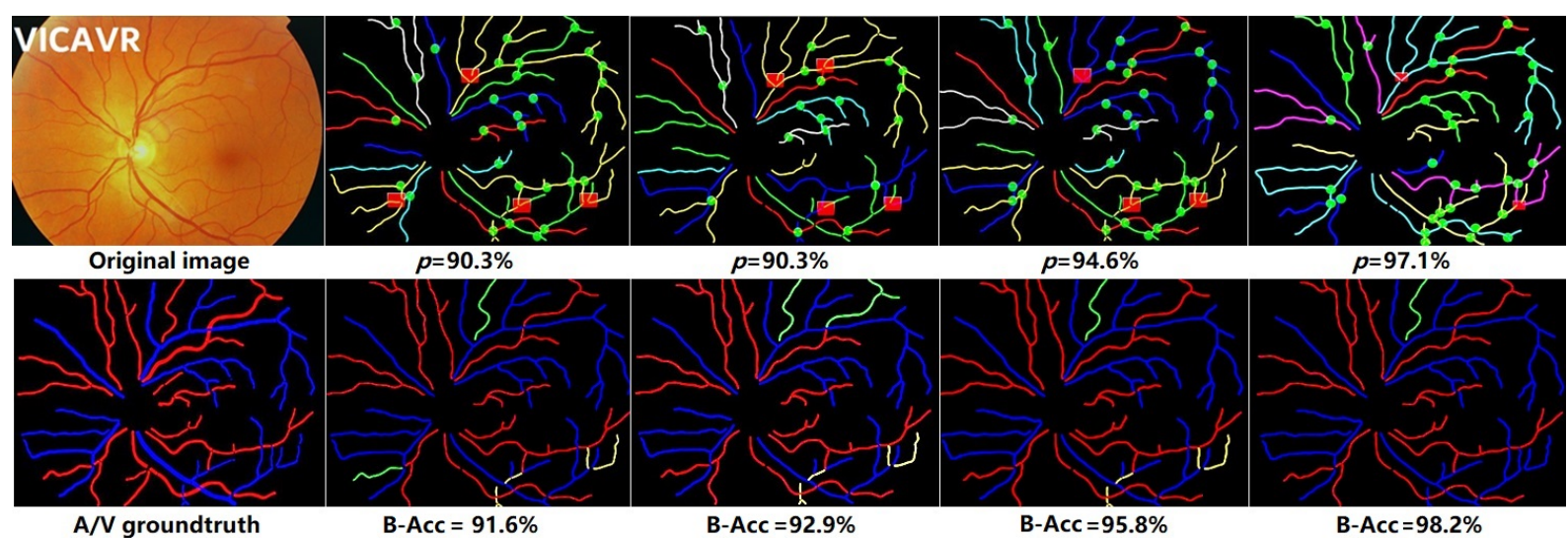

Fig. 10: The topology estimation (top row) and A/V classification (bottom row) results of the proposed method with and without removing different types of features for the representation of the pixels of interest. From second to last column: intensity-based features removed, orientation-based features removed, diameter-based features removed, and all features used.

TABLE VIII: Topology estimation and A/V classification results $(Q / B-A c c)$ of the proposed method with and without removing different types of features for the representation of the pixels of interest.

\begin{tabular}{lccccc}
\hline & INSPIRE & DRIVE & VICAVR & WIDE & IOSTAR \\
\hline All features used & $\mathbf{9 5 . 1 \%} / \mathbf{9 6 . 4 \%}$ & $\mathbf{9 1 . 1 \%} / \mathbf{9 3 . 5 \%}$ & $\mathbf{9 3 . 8 \%} / \mathbf{9 4 . 6 \%}$ & $\mathbf{9 4 . 9 \% / 9 5 . 2 \%}$ & $\mathbf{9 5 . 1 \%} /-$ \\
\hline Diameter-based features removed & $94.2 \% / 94.4 \%$ & $89.1 \% / 91.5 \%$ & $92.0 \% / 93.3 \%$ & $93.4 \% / 94.5 \%$ & $94.1 \% /-$ \\
Orientation-based features removed & $92.9 \% / 93.2 \%$ & $86.8 \% / 88.9 \%$ & $89.9 \% / 91.8 \%$ & $92.5 \% / 93.7 \%$ & $93.0 \% /-$ \\
Intensity-based features removed & $91.0 \% / 92.1 \%$ & $85.1 \% / 88.5 \%$ & $88.3 \% / 90.6 \%$ & $91.9 \% / 92.1 \%$ & $92.2 \% /-$ \\
\hline
\end{tabular}
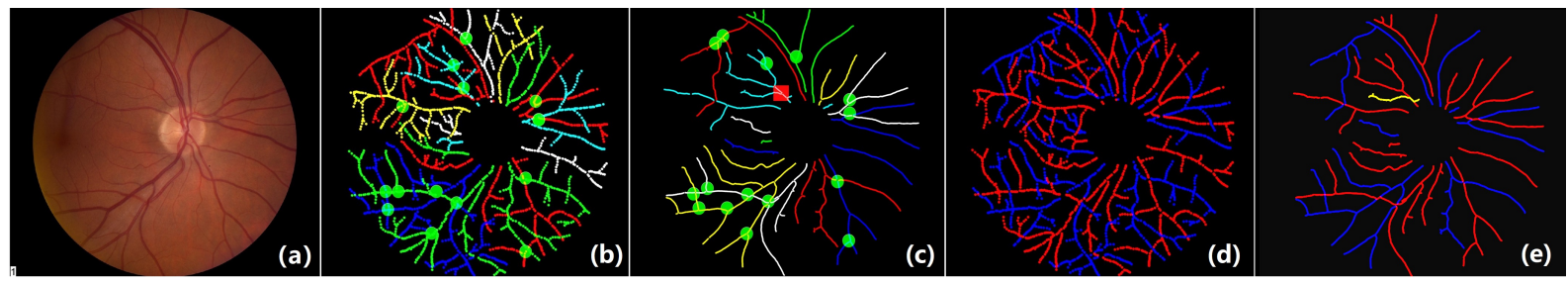

Fig. 11: Illustrative results of the proposed method for vessel topology estimation and $\mathrm{A} / \mathrm{V}$ classification applied on automatic and manual segmentations. (a) A randomly selected image from INSPIRE; (b)-(c) the topology estimation results applied to manual and automatic segmentations; (d)-(e) A/V classification results on manual and automatic segmentations.

TABLE IX: The performance of the proposed method for vessel topology estimation and A/V classification on automatic and manual segmentations at the node-level and vessel centerline pixel-level over the INSPIRE dataset.

\begin{tabular}{l|ccc||cccc|c}
\hline \hline & \multicolumn{3}{|c|}{ vessel segmentation } & \multicolumn{4}{c|}{ topology estimation } & \multicolumn{1}{c}{ A/V classification } \\
\cline { 2 - 9 } & recall & precision & FDR & \# nodes & \# TP & Q & accuracy & $B$-Acc \\
\hline manual segmentation & - & - & - & 2776 & 2642 & $95.1 \%$ & $97.5 \%$ & $96.4 \%$ \\
automatic segmentation & $83.8 \%$ & $82.1 \%$ & 0.071 & 2480 & 2321 & $93.5 \%$ & $88.9 \%$ & $87.7 \%$ \\
\hline
\end{tabular}

Figure 11 shows that the proposed method has produced perfect topology estimation and $\mathrm{A} / \mathrm{V}$ classification results without any error on manual vessel annotations of an image randomly selected from the INSPIRE dataset. While only one node was incorrectly identified, indicated by the red rectangle in Fig. 11. (c), and one vessel segment was incorrectly classified on the automatic segmented vessels, indicated by the yellow line in Fig. 11(e), since a tiny vessel was mis-detected and leads to the generation of a partly incorrect vessel graph that causes further errors in the vessel topology reconstructions and A/V classifications.

In addition, the percentage of nodes that were correctly identified ( $Q$ score) and accuracy of the topology reconstruc- tion at blood vessel centerline pixel-level were calculated. As can be observed in TABLE IX, the proposed topology estimation method achieved a similar $Q$ score on automatic and manual segmentations: $93.5 \%$ and $95.1 \%$, respectively. However, it yielded significantly lower scores of accuracy and $B$-Acc, by $8.6 \%$ and $8.7 \%$, respectively. As aforementioned, the accuracy and B-Acc were calculated at centerline-level. The automated method achieved $82.1 \%$ and $83.8 \%$ in precision and recall, respectively, in comparison with the manual segmentation. This is because a large portion of tiny vessels were mis-segmented.

However, the obtained $Q$ score implies that the proposed method is able to correctly identify most of the nodes in 
automatically detected vessels. These results show that our topology estimation and its subsequent $\mathrm{A} / \mathrm{V}$ classification, are relatively robust in terms of applying to either automatic or manual vessel segmentation results at node-level, while its overall performance (pixel-level) is still dependent on the completeness and accuracy of the vessel segmentation.

\section{CONCLUSions AND Future Work}

Automated identification of the anatomical connectivity of different blood vessels, and classification of those blood vessels into arteries and veins, respectively, are essential for the automated assessment of vascular changes.

In this paper, we have proposed a novel artery/vein classification method based on vascular topological characteristics. Our framework combines graph-theoretic methods with DOS to accurately analyse the retinal vasculature. The concept of DOS in this work was successfully adapted to formalize the topology estimation and $\mathrm{A} / \mathrm{V}$ classification as a pairwise clustering problem. The core issues then go to work on graph generation and edge weight definition. They have been achieved through image segmentation, skeletonization and identification of significant nodes. The latter is defined as the inverse Euclidean distance between the two end points of an edge in the feature space, where each node is represented as a 23 dimensional feature vector about intensity, orientation, curvature, diameter and entropy. The significance of our method is that it is capable of classifying the whole vascular network, and does not restrict itself to specific regions of interest.

The proposed method has accurately reconstructed the vascular topology and classified the blood vessel types into arteries and veins on five publicly accessible retinal datasets. The results show that our method produces better results when compared with the state-of-the-art topology estimation and $\mathrm{A} / \mathrm{V}$ classification ones. It can be expected that the proposed method could be a powerful tool for analyzing vasculature for better management of a wide spectrum of vascular-related diseases.

In addition, we have manually labeled the blood vessel topology for four publicly-available retinal datasets (INSPIRE, VICAVR, DRIVE, and IOSTAR), and these annotations have been released for public access to help other researchers in the community in performing research and development on the same and related topics. For future work, we will test our method on other retinal datasets (e.g., RITE [47]) and neuronal datasets (e.g., DIADEM [48]), and refine the initial graph generation to improve the accuracy of the retinal topology reconstruction.

\section{REFERENCES}

[1] M. Niemeijer, X. Xu, A. Dumitrescu, B. van Ginneken, J. Folk, and M. Abràmoff, "Automated measurement of the arteriolar-to-venular width ratio in digital color fundus photographs," IEEE Trans. Med. Imaging, vol. 30, no. 11, pp. 1941-1950, 2011.

[2] J. Xie, Y. Zhao, Y. Zheng, P. Su, J. Liu, and Y. Wang, "Retinal vascular topology estimation via dominant sets clustering," in Proc. ISBI, 2018, pp. $1458-1462$.

[3] S. Vázquez, B. Cancela, N. Barreira, and M. Saez, "Improving retinal artery and vein classification by means of a minimal path approach," Mach. Vis. Appl., vol. 24, no. 5, pp. 919-930, 2013.
[4] T. Na, J. Xie, Y. Zhao, Y. Zhao, Y. Liu, Y. Wang, and J. Liu, "Retinal vascular segmentation using superpixel-based line operator and its application to vascular topology estimation." Medical physics, vol. 45 7, pp. 3132-3146, 2018.

[5] R. Estrada, C. Tomasi, S. Schmidler, and S. Farsiu, "Tree topology estimation," IEEE Trans. Pattern Anal. Mach. Intell., vol. 37, no. 8, pp. 1688-1701, 2015.

[6] J. Xie, Y. Zhao, Y. Liu, P. Su, Y. Zhao, J. Cheng, Y. Zheng, and J. Liu, "Topology reconstruction of tree-like structure in images via structural similarity measure and dominant set clustering," in Proc. CVPR, 2019, pp. 8505-8513.

[7] Y. Zhao, J. Zhao, J. Yang, Y. Liu, Y. Zhao, Y. Zheng, L. Xia, and Y. Wang, "Saliency driven vasculature segmentation with infinite perimeter active contour model," Neurocomputing, vol. 259, pp. 201209, 2017.

[8] Y. Zhao, Y. Zheng, Y. Liu, Na, and Y. W. J. Liu, "Automatic 2d/3d vessel enhancement in multiple modality images using a weighted symmetry filter," IEEE Trans. Med. Imaging, pp. 1-1, 2017.

[9] B. Dashtbozorg, A. M. Mendonça, and A. Campilho, "An automatic graph-based approach for artery/vein classification in retinal images," IEEE Trans. Image Processing, vol. 23, no. 3, pp. 1073-1083, 2014.

[10] V. Joshi, J.Reinhardt, M. Garvin, and M. Abràmoff, "Automated method for identification and artery-venous classification of vessel trees in retinal vessel networks," PLOS ONE, vol. 9, no. 2, pp. 1-12, 2014.

[11] Y. Zhao, J. Xie, P. Su, Y. Zheng, Y. Liu, J. Cheng, and J. Liu, "Retinal artery and vein classification via dominant sets clustering based vascular topology estimation," in Proc. MICCAI, 2018, pp. 109-118.

[12] M. Pavan and M. Pelillo, "Dominant sets and hierarchical clustering," in Proc. ICCV, 2003, pp. 362-369.

[13] E. Zemene and M. Pelillo, "Interactive image segmentation using constrained dominant sets," in Proc. ECCV, 2016, pp. 278-294.

[14] E. Zemene, L. Alemu, and M. Pelillo, "Dominant sets for "constrained" image segmentation," IEEE Trans. Pattern Anal. Mach. Intell., 2018.

[15] Y. Zhao, Y. Zheng, Y. Liu, J. Yang, Y. Zhao, D. Chen, and Y. Wang, "Intensity and compactness enabled saliency estimation for leakage detection in diabetic and malarial retinopathy," IEEE Trans. Med. Imaging, vol. 36, no. 1, pp. 51-63, 2017.

[16] G. Azzopardi, N. Strisciuglio, M. Vento, and N. Petkov, "Trainable COSFIRE filters for vessel delineation with application to retinal images," Med. Image Anal., vol. 19, pp. 46-57, 2015.

[17] Y. Zhao, Y. Liu, X. Wu, S. Harding, and Y. Zheng, "Retinal vessel segmentation: An efficient graph cut approach with retinex and local phase," PLOS ONE, vol. 10, p. e0122332, 2015.

[18] M. Fraz, P. Remagnino, A. Hoppe, B. Uyyanonvara, A. R. Rudnicka, C. G. Owen, and S. A. Barman, "Blood vessel segmentation methodologies in retinal images - a survey," Comput. Meth. Prog. Bio., vol. 108, pp. 407-433, 2012.

[19] J. Soares and M. Cree, "Retinal vessel segmentation using the 2D Gabor wavelet and supervised classification," IEEE Trans. Med. Imaging, vol. 25, pp. 1214-1222, 2006.

[20] M. Martínez-Pérez, A. Hughes, A. Stanton, S. Thom, N. Chapman, A. Bharath, and K. Parker, "Retinal vascular tree morphology: a semiautomatic quantification," IEEE Trans. Biomed. Engineering, vol. 49, no. 8, pp. 912-917, 2002

[21] C. Kondermann, D. Kondermann, and M. Yan, "Blood vessel classification into arteries and veins in retinal images," in Proc. SPIE Medical Imaging, 2007, p. 651247.

[22] M. Niemeijer, B. Ginneken, and M. Abramoff, "Automatic classification of retinal vessels into arteries and veins," in Proc. SPIE Medical Imaging, vol. 7260, 2009, pp. 72601F-1-72 601F-8.

[23] Q. Mirsharif, F. Tajeripour, and H. Pourreza, "Automated characterization of blood vessels as arteries and veins in retinal images," Comp. Med. Imag. and Graph., vol. 37, no. 7-8, pp. 607-617, 2013.

[24] D. Relan, T. MacGillivray, L. Ballerini, and E. Trucco, "Retinal vessel classification: Sorting arteries and veins," in Proc. EMBC, 2013, pp. 7396-7399.

[25] F. Girard and F. Cheriet, "Artery/vein classification in fundus images using CNN and likelihood score propagation," in Proc. IEEE Global Conf. on Sig. and Info. Process., 2017, pp. 720-724.

[26] F. Huang, B. Dashtbozorg, and B. H. Romeny, "Artery/vein classification using reflection features in retina fundus images," Mach. Vis. Appl., vol. 29 , no. 1, pp. 23-34, 2018.

[27] K. Rothaus, X. Jiang, and P. Rhiem, "Separation of the retinal vascular graph in arteries and veins based upon structural knowledge," Image Vision Comput., vol. 27, no. 7, pp. 864-875, 2009. 
[28] V. Joshi, M. Garvin, J. Reinhardt, and M. Abramoff, "Automated method for the identification and analysis of vascular tree structures in retinal vessel network," in Proc. SPIE Medical Imaging, 2011, p. 79630I.

[29] R. Estrada, M. J. Allingham, P. S. Mettu, S. W. Cousins, C. Tomasi, and S. Farsiu, "Retinal artery-vein classification via topology estimation," IEEE Trans. Med. Imaging, vol. 34, no. 12, pp. 2518-2534, 2015.

[30] X. Lyu, Q. Yang, S. Xia, and S. Zhang, "Construction of retinal vascular trees via curvature orientation prior," in Proc. BIBM, 2016, pp. 375-382.

[31] J. De, L. Cheng, X. Zhang, F. Lin, H. Li, K. Ong, W. Yu, Y. Yu, and S. Ahmed, "A graph-theoretical approach for tracing filamentary structures in neuronal and retinal images," IEEE Trans. Med. Imaging, vol. 35, no. 1, pp. 257-72, 2016.

[32] J. De, X. Zhang, F. Lin, and L. Cheng, "Transduction on directed graphs via absorbing random walks," IEEE Trans. Pattern Anal. Mach. Intell., vol. 40, no. 7, pp. 1770-1784, 2018.

[33] J. Cheng, J. Liu, Y. Xu, F. Yin, D. Wong, N. Tan, D. Tao, C. Cheng, T. Aung, and T. Wong, "Superpixel classification based optic disc and optic cup segmentation for glaucoma screening," IEEE Trans. Med. Imaging, vol. 32, no. 6, pp. 1019-1032, 2013.

[34] Y. Zhao, L. Rada, K. Chen, S. Harding, and Y. Zheng, "Automated vessel segmentation using infinite perimeter active contour model with hybrid region information with application to retinal images," IEEE Trans. Med. Imaging, vol. 34, no. 9, pp. 1797-1807, 2015.

[35] P. Bankhead, J. McGeown, and T. Curtis, "Fast retinal vessel detection and measurement using wavelets and edge location refinement," PLOS ONE, vol. 7, p. e32435, 2009.

[36] M. Pavan and M. Pelillo, "Dominant sets and pairwise clustering," IEEE Trans. Pattern Anal. Mach. Intell., vol. 29, no. 1, pp. 167-172, 2007.

[37] J. Zhang, B. Dashtbozorg, E. J. Bekkers, J. P. W. Pluim, R. Duits, and B. M. ter Haar Romeny, "Robust retinal vessel segmentation via locally adaptive derivative frames in orientation scores," IEEE Trans. Med. Imaging, vol. 35, pp. 2631-2644, 2016.

[38] J. Staal, M. Abràmoff, M. Niemeijer, M. Viergever, and B. van Ginneken, "Ridge-based vessel segmentation in color images of the retina," IEEE Transactions on Medical Imaging, vol. 23, pp. 501-509, 2004.

[39] P. Guimarães, J. Wigdahl, and A. Ruggeri, "A fast and efficient technique for the automatic tracing of corneal nerves in confocal microscopy," Transl. Vis. Sci. Techn., vol. 5, p. Article 7, 2016.

[40] C. Muramatsu, Y. Hatanaka, T. Iwase, T. Hara, and H. Fujita, "Automated selection of major arteries and veins for measurement of arteriolar-to-venular diameter ratio on retinal fundus images," Comput. Med. Imag. Grap., vol. 35 6, pp. 472-80, 2011.

[41] E. Pellegrini, G. Robertson, T. J. MacGillivray, J. van Hemert, G. Houston, and E. Trucco, "A graph cut approach to artery/vein classification in ultra-widefield scanning laser ophthalmoscopy," IEEE Trans. Med. Imaging, vol. 37, pp. 516-526, 2018.

[42] T. Qureshi, M. Habib, A. Hunter, and B. Al-Diri, "A manually-labeled, artery/vein classified benchmark for the drive dataset," in Proc. CBMS, 2013, pp. 485-488.

[43] K. P. Murphy, Y. Weiss, and M. I. Jordan, "Loopy belief propagation for approximate inference: An empirical study," in Proc. ICUAL, 1999, pp. $467-475$.

[44] D. Zhou and B. Schölkopf, "Learning from labeled and unlabeled data on a directed graph," in Proc. ICML, 2005, pp. 1036-1043.

[45] H. Cai, X.and Wang, H. Huang, and C. Ding, "Simultaneous image classification and annotation via biased random walk on tri-relational graph," in Proc. ECCV, 2012, pp. 823-836.

[46] L. Cheng, J. De, X. Zhang, F. Lin, and H. Li, "Tracing retinal blood vessels by matrix-forest theorem of directed graphs," in Proc. MICCAI, 2014, pp. 626-633.

[47] Q. Hu, M. D. Abramoff, and M. K. Garvin, "Automated construction of arterial and venous trees in retinal images," Journal of Med. Imaging, vol. 2, p. 044001, 2015.

[48] T. A. Gillette, K. M. Brown, and G. A. Ascoli, "The DIADEM metric: Comparing multiple reconstructions of the same neuron," Neuroinfo., vol. 9, no. 2-3, pp. 233-245, 2011 
2019-07-03

\section{Retinal vascular network topology reconstruction and artery/vein classification via dominant set clustering}

\section{Zhao, Yitian}

IEEE

Zhao Y, Xie J, Zhang H, et al., (2020) Retinal vascular network topology reconstruction and artery/vein classification via dominant set clustering. IEEE Transactions on Medical Imaging, Volume 39, Issue 2, February 2020, pp. 341-356

https://doi.org/10.1109/TMI.2019.2926492

Downloaded from Cranfield Library Services E-Repository 